COMMUN. IN PARTIAL DIFFERENTIAL EQUATIONS, 18(3\&4), 453-490 (1993)

\title{
SPINODAL DECOMPOSITION FOR THE CAHN-HILLIARD EQUATION
}

\author{
Christopher P. Grant \\ Department of Mathematics \\ University of Utah \\ Salt Lake City, Utah 84112
}

\begin{abstract}
The Cahn-Hilliard equation is a fourth-order parabolic partial differential equation that is one of the leading models for the study of phase separation in isothermal, isotropic, binary mixtures, such as molten alloys. When a spatially homogeneous alloy is rapidly quenched in a physical experiment, a fine-grained decomposition into two distinct phases is frequently observed; this phenomenon is known as spinodal decomposition. A simple linear analysis about an unstable homogeneous equilibrium of the one-dimensional Cahn-Hilliard equation gives heuristic evidence that most solutions that start with initial data near such an equilibrium exhibit a behavior corresponding to spinodal decomposition. In this paper we formulate this conjecture in a mathematically precise way, using geometric and measure-theoretic techniques, and prove its validity. We believe that this is the first rigorous treatment of this phenomenon.
\end{abstract}

\section{Introduction}

When a molten binary alloy is rapidly quenched to a lower temperature, the sample may become inhomogeneous very quickly, decomposing into a fine- 


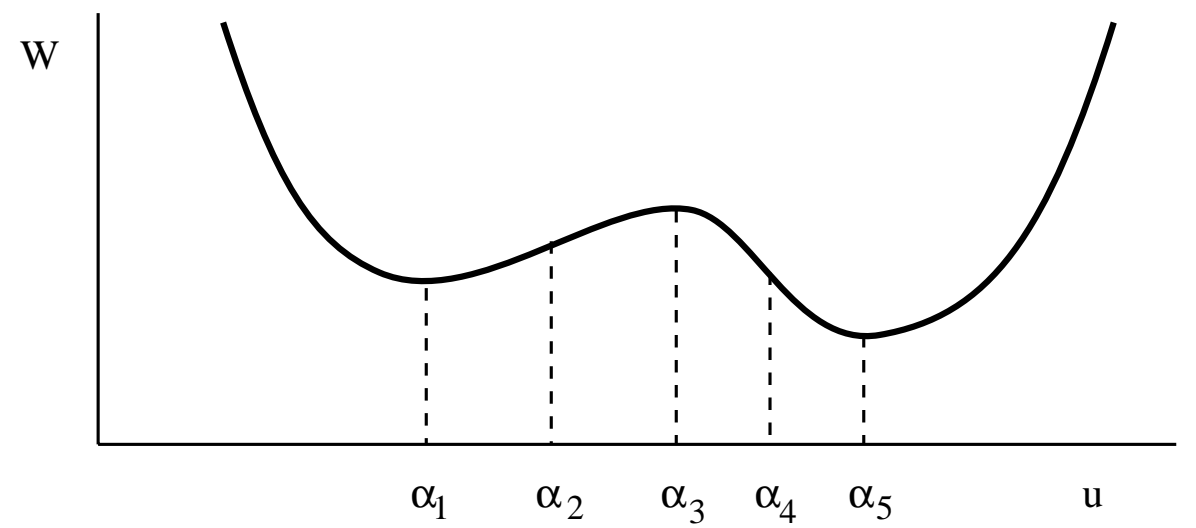

FIG. 1: Bulk free energy density.

grained mixture of particles in different phases. For example, micrographs in [3] and [4] clearly show a fine-grained structure, with a characteristic length scale, appearing in a variety of materials under such circumstances. This phenomenon is known as spinodal decomposition.

One of the leading models devised for the study of spinodal decomposition is the Cahn-Hilliard equation

$$
\begin{array}{rl}
\frac{\partial u}{\partial t}=-\Delta\left(\varepsilon^{2} \Delta u-W^{\prime}(u)\right) & x \in \Omega \\
\frac{\partial u}{\partial \nu}=\frac{\partial \Delta u}{\partial \nu}=0 & x \in \partial \Omega
\end{array}
$$

where $\Omega$ is a bounded open subset of $\mathbf{R}^{n}$ with sufficiently smooth boundary, $\nu$ is the unit outward normal, $\varepsilon$ is a small positive parameter, and $W$ is a $C^{5}$ function qualitatively similar to the one depicted in Figure 1. The interval where $W^{\prime \prime}<0$ is known as the spinodal region. The variable $u$ represents the concentration of one of the two components of the alloy, so $\int_{\Omega} u d x$ represents the total mass of that component. Note that the boundary conditions imply that mass is conserved, since

$$
\begin{aligned}
\frac{d}{d t} \int_{\Omega} u d x & =\int_{\Omega} \frac{\partial u}{\partial t} d x=\int_{\Omega}\left[-\Delta\left(\varepsilon^{2} \Delta u-W^{\prime}(u)\right)\right] d x \\
& =-\int_{\partial \Omega}\left[\frac{\partial}{\partial \nu}\left(\varepsilon^{2} \Delta u-W^{\prime}(u)\right)\right] d s=0 .
\end{aligned}
$$

In 1893 van der Waals [24] first suggested that the functional 


$$
\int_{\Omega}\left[W(u)+\frac{\varepsilon^{2}}{2}|\nabla u|^{2}\right] d x
$$

was a good model for the free energy of binary mixtures. Over 60 years later Cahn and Hilliard [5] [2] rediscovered this functional and derived (1) as the evolution equation induced by (2) through Fick's law of diffusion. They performed a linear analysis of (1) and argued that the results of this analysis suggested what we shall call the Principle of Spinodal Decomposition: Most solutions to the Cahn-Hilliard equation that start with initial data near a fixed constant in the spinodal region exhibit fine-grained decomposition. Since this conjecture agrees with the outcome of physical experiments, the CahnHilliard equation has been accepted as a meaningful model of the dynamics of phase transitions.

In the last 30 years the Cahn-Hilliard equation has been the subject of much study. For example, existence and uniqueness theorems for solutions of (1) in various function spaces have been proven by, among others, Elliott and Zheng [7], Nicolaenko and Scheurer [17], Rankin [20], and Temam [23]. Carr, Gurtin, and Slemrod [6], Modica [15], Novick-Cohen and Segel [18], and Zheng [25], have results about steady-state solutions of (1); these results are important in understanding the asymptotic behavior of arbitrary solutions to (1) because the Cahn-Hilliard equation is a dissipative system (see, e.g., [23]), so each of its solutions approaches a time-independent solution (or possibly a collection of such solutions) as $t$ becomes large.

In spite of this work, several aspects of the Cahn-Hilliard equation have not received a rigorous mathematical treatment. In particular, the Principle of Spinodal Decomposition stated above has neither been formulated with mathematical precision nor been proven to be valid. In this paper, we make some progress towards filling in this gap by stating and proving a number of formulations of the Principle of Spinodal Decomposition when $\Omega$ is onedimensional.

We will proceed as follows. In Section 2 a linear analysis of the CahnHilliard equation will be performed and it will be explained why this analysis suggests that spinodal decomposition occurs. Much of the work necessary to justify this conjecture does not make use of the special structure of the Cahn-Hilliard equation, so this part, which can be found in Section 3, will be performed in the framework of an ordinary differential equation in an abstract Hilbert space, using the theory of analytic semigroups. This theory can be used to show the existence of a pseudo-unstable manifold, an invariant manifold consisting of the fastest-growing solutions in a neighborhood of a fixed equilibrium. Here we obtain this manifold as a fairly easy consequence of some 
results on invariant manifolds for maps by Hirsch, Pugh, and Shub [12]. This manifold could also be found as a submanifold of the unstable manifold if the latter is finite-dimensional.

What is of more interest than the pseudo-unstable manifold itself is the flow nearby. We will construct an open positively-invariant neighborhood of the manifold that contains positive semiorbits that have similar long-time behaviors and estimate the location of the boundaries of this set near the equilibrium. This is reminiscent of some work found in Chapter 5 of Henry [11], where he defines sets that have certain invariance properties in a neighborhood of an equilibrium, but our approaches are quite different, and there does not seem to be an easy way to use his particular methods to obtain the results we need for spinodal decomposition. Additionally, our methods avoid the need to renorm the space, so we preserve the original Hilbert space structure. This fact allows us to translate the geometric estimates on the boundaries of the invariant set into statements about the measure of the set, with respect to any (non-degenerate) Gaussian measure. (A Gaussian measure is a generalization of the one-dimensional normal distribution.)

In Section 4, the special properties of the one-dimensional Cahn-Hilliard equation will be taken into account; in particular, the periodicity and amplitude of $\omega$-limit points of orbits making up a one-dimensional pseudo-unstable manifold will be described in some detail. This information is sufficient, in combination with the abstract results of Section 3, to show that the Principle of Spinodal Decomposition holds in one dimension. Our main results can be roughly summarized as follows:

1. Sufficiently small perturbations of a generic unstable homogeneous equilibrium that are not orthogonal to a particular direction generate solutions to the Cahn-Hilliard equation which exhibit spinodal decomposition.

2. Near a generic unstable homogeneous equilibrium, there is a set of initial data whose boundary satisfies a simple power law such that solutions originating in this set exhibit spinodal decomposition. The particular power governing the boundary is dependent upon the size of a particular spectral gap of the linearized Cahn-Hilliard equation.

3. Given a generic unstable homogeneous equilibrium and a nondegenerate Gaussian measure centered at this equilibrium, as the covariance of the measure shrinks to 0 through rescaling, the measure of the set of initial data that produces solutions exhibiting spinodal decomposition 
approaches 1 at an asymptotic rate dependent on the spectral gap mentioned above.

These results constitute Theorems 6, 7, and 8, respectively. Because of their technical nature, the precise statement of these theorems will be postponed to Section 4.

\section{Linear Analysis}

\subsection{The Dispersion Relation}

At this point, let us be more precise about the requirements for the nonlinearity $W$. Although Cahn and Hilliard originally used a function $W$ which approached infinity at finite values of $u$, we will follow the convention of most of the recent literature and assume that $W$ is defined for all real numbers. We will also assume that there exist real numbers $\alpha_{1}<\alpha_{2}<\alpha_{3}<\alpha_{4}<\alpha_{5}$ such that $W$ is strictly decreasing on $\left(-\infty, \alpha_{1}\right]$ and on $\left[\alpha_{3}, \alpha_{5}\right]$, strictly increasing on $\left[\alpha_{1}, \alpha_{3}\right]$ and on $\left[\alpha_{5}, \infty\right)$, concave up on $\left(-\infty, \alpha_{2}\right) \cup\left(\alpha_{4}, \infty\right)$, and concave down on $\left(\alpha_{2}, \alpha_{4}\right)$. (See Figure 1.)

Now consider the Cahn-Hilliard equation when $\Omega=[0,1]$ :

$$
\begin{array}{rl}
u_{t}=-\left(\varepsilon^{2} u_{x x}-W^{\prime}(u)\right)_{x x} & x \in(0,1) \\
u_{x}=u_{x x x}=0 & x \in\{0,1\} .
\end{array}
$$

If the mass $M$ is in the spinodal region then $\beta^{2} \stackrel{\text { def }}{=}-W^{\prime \prime}(M)>0$. Linearizing (3) about $u \equiv M$ gives

$$
\begin{array}{rl}
u_{t}=-\varepsilon^{2} u_{x x x x}-\beta^{2} u_{x x} & x \in(0,1) \\
u_{x}=u_{x x x}=0 & x \in\{0,1\} .
\end{array}
$$

The eigenfunctions of the linear operator on the right-hand side of the first line of (4), subject to the boundary conditions in the second line, are

$$
\{\cos n \pi x: n=0,1,2, \ldots\}
$$

Substituting $u=a_{n} \cos n \pi x$ into (4), where $a_{n}$ is a function of $t$, gives

$$
a_{n}(t)=a_{n}(0) \exp \left(\lambda_{n} t\right)
$$

where

$$
\lambda_{n}=(n \pi)^{2}\left[\beta^{2}-\varepsilon^{2}(n \pi)^{2}\right] .
$$


For $0<n<\beta /(\varepsilon \pi), \lambda_{n}>0$, so the corresponding Fourier modes grow as time progresses in the linearized equation (4), while the other modes remain steady or shrink. Thus, for $\varepsilon<\beta / \pi$ spatially homogeneous equilibria in the spinodal region are linearly unstable. Elementary calculus implies that the maximum of $\lambda_{n}$ over all $n \in \mathbf{R}$ occurs when $n=\beta /(\varepsilon \pi \sqrt{2})$, so the one or two integer values of $n$ whose squares are nearest to $\beta^{2} /\left(2 \varepsilon^{2} \pi^{2}\right)$ correspond to the modes that grow the most rapidly under (4). It is not hard to see that the largest eigenvalue of the linearized operator is simple except when $\lambda_{n}=\lambda_{n+1}$ for some $n \in \mathbf{N}$. An easy calculation shows that this holds when

$$
\varepsilon=\frac{\beta}{\pi \sqrt{2 n^{2}+2 n+1}} .
$$

In particular, there is generically a unique fastest-growing mode. Also, the wavelength of this mode is $O(\varepsilon)$, so if $\varepsilon$ is small then the wavelength of the fastest-growing mode is small, also.

To understand the implications of the linearized analysis done in the preceding section, consider the behavior of the linear system of ordinary differential equations

$$
\frac{d}{d t}\left(\begin{array}{l}
u_{1} \\
u_{2}
\end{array}\right)=\left(\begin{array}{cc}
\lambda_{+} & 0 \\
0 & \lambda_{-}
\end{array}\right)\left(\begin{array}{l}
u_{1} \\
u_{2}
\end{array}\right),
$$

where $\lambda_{+}>\lambda_{-}>0$. The fastest-growing solutions of this linear system lie completely on the $u_{1}$-axis. Each of the other orbits is tangent to the $u_{2}$-axis and lies on a curve of the form

$$
u_{1}=C u_{2}^{\lambda_{+} / \lambda_{-}}
$$

for some constant $C$. Consider a small circular neighborhood of the origin and the forward semiorbits of (6) that begin there. Most of these semiorbits eventually run nearly parallel to the fastest-growing solution and stay relatively close to it. Thus, in some sense, the fastest-growing solution dominates most orbits starting near the origin.

Now if the solutions to the Cahn-Hilliard equation behave in an analogous fashion then the fastest-growing mode should dominate the behavior of most solutions with initial values that are small perturbations from a constant. Thus, the evolution of a typical small perturbation from the homogeneous steady-state might be as depicted in the three cross-sections in Figure 2. Somewhat independently of the initial data, the characteristic periodic structure of the fastest-growing mode would emerge. In the next two sections, we show that these heuristics are correct. 


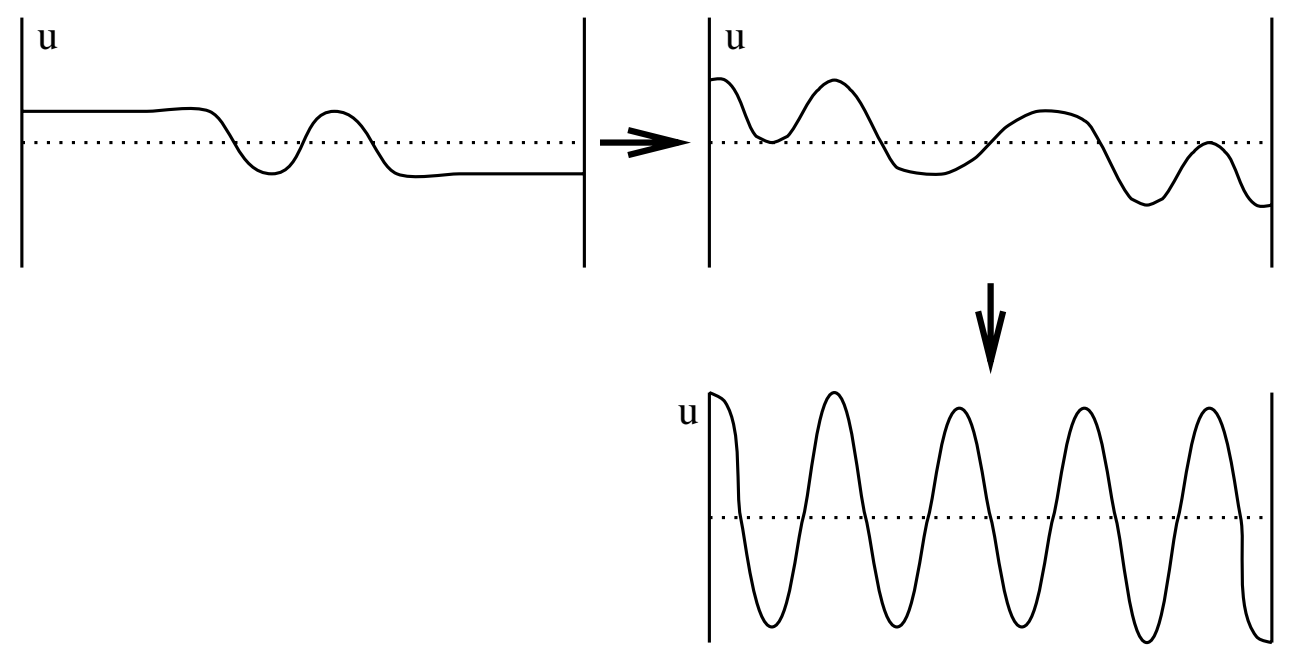

FIG. 2: Typical evolution sequence for the Cahn-Hilliard equation.

\section{Evolution in a Hilbert Space}

\subsection{The Abstract Setting}

Consider an evolution equation that can be represented in the form

$$
\begin{gathered}
\frac{d}{d t} u(t)=A u(t)+f(u(t)) \quad t>0 \\
u(0)=u_{0},
\end{gathered}
$$

where $u(t)$ is an element of a Hilbert space $X$. Suppose the following hold.

1. The operator $-A$ is a sectorial operator on $X$, and $S(t)$ is the analytic semigroup generated by $A$.

2. For some $\alpha \in[0,1), f$ maps $X^{\alpha}$ into $X$, where $X^{\alpha} \equiv \mathcal{D}\left(\left(-A_{1}\right)^{\alpha}\right)$ is a Hilbert space with an inner product equivalent to the graph norm, and $A_{1}=A-a_{1}$ for some $a_{1}$ such that $\operatorname{Re} \sigma\left(A_{1}\right)<0$. Both the inner product and the induced norm will be identified with a subscript $\alpha$.

3. The map $f: X^{\alpha} \rightarrow X$ is $C^{1}$ and satisfies $f(0)=0$ and $D f(0)=0$.

4. The operator $A$ induces a decomposition of $X, X=X^{-} \oplus X^{+}$, such that

(a) $X^{-}$and $X^{+}$are invariant under $A$;

(b) $X^{-}$and $X^{+}$are orthogonal with respect to the inner product on $X^{\alpha}$ 
(c) $X^{+}$is finite-dimensional and $X^{+} \subset \mathcal{D}(A)$;

(d) and $\operatorname{Re}\left(\sigma\left(A^{-}\right)\right)<a<b<\operatorname{Re}\left(\sigma\left(A^{+}\right)\right)$, where $A^{-}: X^{-} \cap \mathcal{D}(A) \rightarrow$ $X^{-}$and $A^{+}: X^{+} \rightarrow X^{+}$are the restrictions of $A$ to $X^{-}$and $X^{+}$, respectively, and $\sigma(L)$ represents the spectrum of the linear operator $L$.

Intuition suggests that there is an invariant manifold for (7) that is tangent to $\mathrm{X}^{+}$at 0 and that, in some sense, controls the forward-time flow of nearby solutions. More precisely, the speed at which nearby solutions move in a direction parallel to the manifold should be much greater than the speed at which they are repelled by the manifold. In the special case when $a<0<b$, this invariant manifold is the unstable manifold whose existence is proved by Henry [11]. In the special case when $\alpha=0$ (but for more general $A$ ) Bates and Jones [1] have an elegant proof for the existence of this pseudo-unstable manifold for arbitrary $a$ and $b$. This special case is also the subject of some powerful results recently obtained by Kening $\mathrm{Lu}$ [14]. Here we obtain the existence of pseudo-unstable manifolds for more general nonlinearities as a consequence of the theory of Hirsch, Pugh, and Shub [12] for invariant manifolds for maps. In order to determine the nature of the flow near such a manifold, we construct estimates on the growth or decay of solutions in the $X^{+}$and $X^{-}$directions similar to those used by Bates and Jones in [1]. In their paper, these estimates imply the existence of invariant cones; since our estimates are not as good for the $\alpha \neq 0$ case, we can only construct pairs of cones that have a weaker invariance property. Nevertheless, these are sufficient for our purposes.

At this point it should be mentioned that the pseudo-unstable manifold is not an inertial manifold if $\sigma\left(A^{-}\right)$intersects the right halfplane, since it will not attract nearby semiorbits, let alone attract them exponentially. Thus, the large body of literature concerning the existence and stability of inertial manifolds (see Temam [23] for a survey of this literature) does not apply here.

\subsection{Growth Estimates Near an Equilibrium}

It will be useful to consider the problem obtained when the nonlinearity $f$ in (7) is modified so that it is very well-behaved outside of a small neighborhood of 0 . We make use of the following technical lemma.

Lemma 1 For any $\varepsilon>0$, there exists $\hat{f}: X^{\alpha} \rightarrow X$ such that $\hat{f}$ is $C^{1}$, is globally Lipschitz continuous with a Lipschitz constant no greater than $\varepsilon$, and agrees with $f$ in some neighborhood of 0 . If, in addition, $\|D f(u)\|_{\mathcal{L}\left(X^{\alpha}, X\right)}=$

$O\left(\|u\|_{\alpha}\right)$ then $\hat{f}$ can be made to agree with $f$ on a ball of radius $\rho$ centered at 0 , where $\rho^{-1}=O\left(\varepsilon^{-1}\right)$ as $\varepsilon \rightarrow 0$. 
Proof. Let $\psi: \mathbf{R} \rightarrow[0,1]$ be a $C^{\infty}$ function that is 1 on $[-1,1]$, that is 0 on $(-\infty,-4] \cup[4, \infty)$, and that satisfies $\left|\psi^{\prime}\right| \leq 1$ on $\mathbf{R}$. Choose $\rho>0$ so small that $\|D f(u)\|_{\mathcal{L}\left(X^{\alpha}, X\right)} \leq \varepsilon / 9$ if $\|u\|_{\alpha} \leq \rho$. The mean value theorem then says that $f$ has a Lipschitz constant less than or equal to $\varepsilon / 9$ on the ball of radius $\rho$ centered at 0 . If $\hat{f}$ is defined by

$$
\hat{f}(u)=\psi\left(\frac{4\|u\|_{\alpha}^{2}}{\rho^{2}}\right) f(u)
$$

then $\hat{f}(u)=f(u)$ for $\|u\|_{\alpha} \leq \rho / 2$ and $\hat{f}(u)=0$ for $\|u\|_{\alpha}>\rho$. For $h \in X^{\alpha}$,

$$
D \hat{f}(u) h=\frac{8}{\rho^{2}} \psi^{\prime}\left(\frac{4\|u\|_{\alpha}^{2}}{\rho^{2}}\right)\langle u, h\rangle_{\alpha} f(u)+\psi\left(\frac{4\|u\|_{\alpha}^{2}}{\rho^{2}}\right) D f(u) h,
$$

SO

$$
\begin{aligned}
\|D \hat{f}(u)\|_{\mathcal{L}\left(X^{\alpha}, X\right)} \leq & \frac{8}{\rho^{2}}\left|\psi^{\prime}\left(\frac{4\|u\|_{\alpha}^{2}}{\rho^{2}}\right)\right|\|u\|_{\alpha}\|f(u)\| \\
& +\left|\psi\left(\frac{4\|u\|_{\alpha}^{2}}{\rho^{2}}\right)\right|\|D f(u)\|_{\mathcal{L}\left(X^{\alpha}, X\right)} \leq \varepsilon
\end{aligned}
$$

if $\|u\|_{\alpha} \leq \rho$. By the mean value theorem, this proves that $\hat{f}$ has a global Lipschitz constant no greater than $\varepsilon$.

It is easy to see that if $\|D f(u)\|_{\mathcal{L}\left(X^{\alpha}, X\right)}=O\left(\|u\|_{\alpha}\right)$, then $\rho$ can be chosen so that $\rho^{-1}=O\left(\varepsilon^{-1}\right)$ as $\varepsilon \rightarrow 0$. This completes the proof of the lemma.

The equation that results from modifying the nonlinearity is

$$
\begin{gathered}
\frac{d}{d t} u(t)=A u(t)+\hat{f}(u(t)) \quad t>0 \\
u(0)=u_{0} .
\end{gathered}
$$

Unless it is explicitly stated otherwise, when solutions, semiorbits, etc. are mentioned, reference is being made to (8). The letters $v$ and $w$ will represent elements of $X^{-}$and $X^{+}$, respectively. Also, where the particular topology used is not specified, implicit reference is being made to the $X^{\alpha}$ topology.

Let $S^{-}(t)$ and $S^{+}(t)$ be the analytic semigroups generated by $A^{-}$and $A^{+}$, respectively. Henry [11] gives the following estimates on these semigroups:

$$
\begin{aligned}
& \left\|S^{+}(t) w\right\|_{\alpha} \leq C_{1} e^{b t}\|w\|_{\alpha}, \quad w \in X^{+}, \quad t \leq 0, \\
& \left\|S^{+}(t) w\right\|_{\alpha} \leq C_{2} e^{b t}\|w\|, \quad w \in X^{+}, \quad t \leq 0, \\
& \left\|S^{-}(t) v\right\|_{\alpha} \leq C_{3} e^{a t}\|v\|_{\alpha}, \quad v \in X^{-} \cap X^{\alpha}, \quad t \geq 0, \\
& \left\|S^{-}(t) v\right\|_{\alpha} \leq C_{4} e^{a t} t^{-\alpha}\|v\|, \quad v \in X^{-}, \quad t \geq 0 \text {. }
\end{aligned}
$$


Solutions of (8) satisfy the variation-of-constants formula (see, e.g., [9])

$$
u(t)=S(t) u_{0}+\int_{0}^{t} S(t-s) \hat{f}(u(s)) d s
$$

for $t \geq 0$. Write $u=v+w$ with $v \in X^{-}$and $w \in X^{+}$, and then project (13) onto these two subspaces to get the pair of integral equations

$$
\begin{aligned}
v(t) & =S^{-}(t) v(0)+\int_{0}^{t} S^{-}(t-s) \hat{f}^{-}(v(s), w(s)) d s \\
w(t) & =S^{+}(t) w(0)+\int_{0}^{t} S^{+}(t-s) \hat{f}^{+}(v(s), w(s)) d s
\end{aligned}
$$

for $t \geq 0$, where $\hat{f}^{-}$and $\hat{f}^{+}$are the projections of $\hat{f}$ onto $X^{-}$and $X^{+}$, respectively. Note that $S^{+}(\tau)$ makes sense for negative $\tau$ since $X^{+}$is finitedimensional, so a change of variable in (15) can be made to get

$$
w(t+\tau)=S^{+}(\tau) w(t)+\int_{0}^{\tau} S^{+}(\tau-s) \hat{f}^{+}(v(t+s), w(t+s)) d s
$$

for $\tau \geq-t$. Taking norms in (14) and (16) and using the estimates in (9) through (12) yields

$$
\begin{aligned}
& \|v(t)\|_{\alpha} \leq\|v(0)\|_{\alpha} C_{3} e^{a t} \\
& \quad+C_{4} \varepsilon \int_{0}^{t}\left(\|v(s)\|_{\alpha}+\|w(s)\|_{\alpha}\right)(t-s)^{-\alpha} e^{a(t-s)} d s
\end{aligned}
$$

for $t \geq 0$, and

$$
\begin{aligned}
& \|w(t+\tau)\|_{\alpha} \leq C_{1}\|w(t)\|_{\alpha} e^{b \tau} \\
& \quad+C_{2} \varepsilon \int_{\tau}^{0}\left(\|v(t+s)\|_{\alpha}+\|w(t+s)\|_{\alpha}\right) e^{b(\tau-s)} d s
\end{aligned}
$$

for $0 \geq \tau \geq-t$

Lemma 2 Let $U:[-t, 0] \rightarrow[0, \infty)$ be continuous. Suppose that for some positive $M$ and $N$,

$$
U(\tau) \leq M U(0)+N \int_{\tau}^{0} U(s) d s
$$

for $-t \leq \tau \leq 0$. Then $U(0) \geq U(-t) e^{-N t} / M$.

Proof. Apply the standard version of Gronwall's inequality to $\widehat{U}:[0, t] \rightarrow$ $[0, \infty)$ defined by $\widehat{U}(\tau)=U(-\tau)$.

The following is a generalization of Gronwall's inequality. Results of this type are essential when dealing with analytic semigroups and fractional spaces, 
and related estimates can be found in Henry [11] and Pazy [19], for example. The proof presented here is different in that it is not of an iterative nature.

Lemma 3 Let $U:[0, t] \rightarrow[0, \infty)$ be continuous, and let $0 \leq \alpha<1$. Suppose that for some positive $M$ and $N$,

$$
U(\tau) \leq M U(0)+N \int_{0}^{\tau}(\tau-s)^{-\alpha} U(s) d s
$$

for $0 \leq \tau \leq t$. Then

$$
U(t) \leq 2 M U(0) \exp \left[(2 N \Gamma(1-\alpha))^{1 /(1-\alpha)} t\right]
$$

Proof. Let $\theta=(2 N \Gamma(1-\alpha))^{1 /(1-\alpha)}$, and let $V(t)=2(M U(0)+\varepsilon) \exp (\theta t)$, for some small $\varepsilon>0$. If $\tau>0$ then

$$
\begin{aligned}
\int_{0}^{\tau}(\tau-s)^{-\alpha} V(s) d s & =V(\tau) \int_{0}^{\tau}(\tau-s)^{-\alpha} e^{-\theta(\tau-s)} d s \\
& =\frac{V(\tau)}{\theta^{1-\alpha}} \int_{0}^{\theta \tau} r^{-\alpha} e^{-r} d r \\
& <\frac{V(\tau)}{\theta^{1-\alpha}} \Gamma(1-\alpha) \\
& =\frac{V(\tau)}{2 N}
\end{aligned}
$$

Thus,

$$
\begin{gathered}
M U(0)+\varepsilon+N \int_{0}^{\tau}(\tau-s)^{-\alpha} V(s) d s \\
<M U(0)+\varepsilon+\frac{V(\tau)}{2} \leq V(\tau),
\end{gathered}
$$

for all $\tau>0$.

Now set

$$
\tau^{*}=\sup \{\tau \in[0, t]: \forall s \in[0, \tau], U(s)<V(s)\}
$$

Substituting $\tau=0$ into (19) gives $U(0) \leq M U(0)$, so $U(0)<V(0)$, which means that this set is not empty. Applying (19) and (20) with $\tau=\tau^{*}$ gives

$$
\begin{aligned}
U\left(\tau^{*}\right) & <M U(0)+\varepsilon+N \int_{0}^{\tau^{*}}\left(\tau^{*}-s\right)^{-\alpha} V(s) d s \\
& \leq V\left(\tau^{*}\right)
\end{aligned}
$$

Since $U$ and $V$ are continuous, the strict inequality $U\left(\tau^{*}\right)<V\left(\tau^{*}\right)$ must mean that $\tau^{*}=t$, so 


$$
U(t)<V(t)=2(M U(0)+\varepsilon) \exp \left[(2 N \Gamma(1-\alpha))^{1 /(1-\alpha)} t\right]
$$

Letting $\varepsilon \rightarrow 0$ gives the stated conclusion.

Now suppose $\|w(s)\|_{\alpha} \geq \mu\|v(s)\|_{\alpha}$ for $0 \leq s \leq t$. Then $v$ can be eliminated from the right-hand side of (18), and an application of Lemma 2 with $U(\tau)=$ $\|w(t+\tau)\|_{\alpha} e^{-b \tau}$ yields

$$
\|w(t)\|_{\alpha} \geq C_{1}^{-1}\|w(0)\|_{\alpha} \exp \left[\left(b-C_{2} \varepsilon\left(1+\mu^{-1}\right)\right) t\right] .
$$

Suppose, on the other hand, that $\|w(s)\|_{\alpha} \leq \mu\|v(s)\|_{\alpha}$ for $0 \leq s \leq t$. Then $w$ can be eliminated from the right-hand side of (17), and an application of Lemma 3 with $U(t)=\|v(t)\|_{\alpha} e^{-a t}$ yields

$$
\|v(t)\|_{\alpha} \leq 2 C_{3}\|v(0)\|_{\alpha} \exp \left[\left(a+\left(2 C_{4} \varepsilon(1+\mu) \Gamma(1-\alpha)\right)^{1 /(1-\alpha)}\right) t\right] .
$$

These two estimates hold for the evolution with the modified nonlinearity $\hat{f}$, but note that they also hold in a neighborhood of 0 for the original nonlinearity. Note also that no $C_{j}$ is in any way dependent on $\varepsilon$ or $\mu$.

\subsection{Invariant Manifolds}

According to Theorem 3.4.4 in [11] the time- $t$ map $T(t)$ induced by (8) is continuously differentiable from $X^{\alpha}$ to $X^{\alpha}$ and the derivative of this map at 0 is $S(t)$. Let $a<\gamma<b$. Then the estimates (9) and (11) imply that for $t$ sufficiently large $S(t)$ is $e^{\gamma t}$-pseudo hyperbolic, using the terminology of Hirsch, Pugh, and Shub [12]. Also, the canonical spectral decomposition of $X^{\alpha}$ corresponding to this pseudo hyperbolic endomorphism is compatible with the decomposition $X^{-} \oplus X^{+}$of $X$.

Lemma 4 For fixed $t$, the global Lipschitz constant of $T(t)-S(t)$ can be made arbitrarily small by making the Lipschitz constant of $\hat{f}$ sufficiently small.

Proof. Because of (11) and (12), and since $X^{+}$is finite-dimensional, there must exist valid estimates of the form

$$
\begin{array}{r}
\|S(t) u\|_{\alpha} \leq C_{5} e^{k t} t^{-\alpha}\|u\| \\
\|S(t) u\|_{\alpha} \leq C_{6} e^{k t}\|u\|_{\alpha} .
\end{array}
$$

Let $u_{1}(t)$ and $u_{2}(t)$ be two solutions of (8). Then by (13) and (23),

$$
\left\|(T(t)-S(t)) u_{1}(0)-(T(t)-S(t)) u_{2}(0)\right\|_{\alpha}
$$




$$
\begin{aligned}
& =\left\|\int_{0}^{t} S(t-s)\left(\hat{f}\left(u_{1}(s)\right)-\hat{f}\left(u_{2}(s)\right)\right) d s\right\|_{\alpha} \\
& \leq C_{5} \varepsilon \int_{0}^{t} \frac{e^{k(t-s)}}{(t-s)^{\alpha}}\left\|u_{1}(s)-u_{2}(s)\right\|_{\alpha} d s .
\end{aligned}
$$

Also by (13), (23), and (24),

$$
\begin{aligned}
\left\|u_{1}(t)-u_{2}(t)\right\|_{\alpha} \leq & C_{6} e^{k t}\left\|u_{1}(0)-u_{2}(0)\right\|_{\alpha} \\
& +C_{5} \varepsilon \int_{0}^{t} \frac{e^{k(t-s)}}{(t-s)^{\alpha}}\left\|u_{1}(s)-u_{2}(s)\right\|_{\alpha} d s .
\end{aligned}
$$

If Lemma 3 is applied to $(26)$ with $U(t)=\left\|u_{1}(t)-u_{2}(t)\right\|_{\alpha} e^{-k t}$, then for $\varepsilon<1$,

$$
\left\|u_{1}(t)-u_{2}(t)\right\|_{\alpha} \leq 2 C_{6} \exp \left(C_{7} t\right)\left\|u_{1}(0)-u_{2}(0)\right\|_{\alpha}
$$

with $C_{7}$ independent of $\varepsilon$. By substituting (27) into (25) it can be seen that there is $C_{8}$ depending on $t$ but not on $\varepsilon, u_{1}(0)$, or $u_{2}(0)$, such that

$$
\left\|(T(t)-S(t)) u_{1}(0)-(T(t)-S(t)) u_{2}(0)\right\|_{\alpha} \leq C_{8} \varepsilon\left\|u_{1}(0)-u_{2}(0)\right\|_{\alpha} .
$$

This completes the proof of the lemma.

Lemma 4 completes the verification of all the hypotheses of Theorem 5.1 and Corollary 5.3 in [12]. Those results imply that if $t$ is sufficiently large and if $\varepsilon$ is sufficiently small then the set

$$
\overline{W^{+}} \stackrel{\text { def }}{=}\left\{u \in X^{\alpha}: \forall n, T(t)^{-n} u \text { exists and }\left\|T(t)^{-n} u\right\|_{\alpha} e^{\gamma n t} \rightarrow 0 \text { as } n \rightarrow \infty\right\}
$$

is the graph of a $C^{1}$ map from $X^{+}$to $X^{-} \cap X^{\alpha}$ that is tangent to $X^{+}$at 0 . In the definition of $\overline{W^{+}}$, it is only required that some backward semiorbit of the map $T(t)$ exists, and that the given estimate holds along it.

This is the pseudo-unstable manifold of the time- $t$ map of (8) (corresponding to the particular decomposition of the space under consideration). A similar set can be defined for the semiflow of (8):

$$
W^{+} \stackrel{\text { def }}{=}\left\{u \in X^{\alpha}: T(t)^{-1} u \text { exists for } t>0,\left\|T(t)^{-1} u\right\|_{\alpha} e^{\gamma t} \rightarrow 0 \text { as } t \rightarrow \infty\right\},
$$

where, as with $\overline{W^{+}}$, the estimate in the definition of $W^{+}$holds along some backward semiorbit. Clearly $W^{+} \subseteq \overline{W^{+}}$. By the proof of Lemma 4, there is a maximum factor by which the norm of a solution $\|u(t)\|_{\alpha}$ can grow within a fixed length of time. This implies that, in fact, $W^{+}=\overline{W^{+}}$, so a pseudounstable manifold exists for the semiflow of (8). The intersection of this with a small neighborhood of 0 gives a local version of this invariant manifold for 
(7), but it might be dependent on the behavior of orbits far from the origin, in which case it will not have a simple characterization in terms of the growth rates of solutions of $(7)$. However, if we restrict the location of the spectral gap $(a, b)$ by assuming that $b>0$ (and $\gamma>0$ ) then the version of $W^{+}$for $(7)$ does not have this pathology, so it will have a characterization in terms of (7) analogous to the characterization of $W^{+}$in terms of (8). As Hirsch, Pugh, and Shub point out in [12], this characterization implies uniqueness.

\subsection{Flow near the Pseudo-unstable Manifold}

\subsubsection{Evolution in Cones}

If $\mu>0$ let

$$
\begin{gathered}
K_{\mu}=\left\{v+w \in X^{\alpha}: \mu\|v\|_{\alpha} \leq\|w\|_{\alpha}\right\}, \\
\gamma_{\mu}^{+}(\varepsilon)=b-C_{2} \varepsilon\left(1+\mu^{-1}\right),
\end{gathered}
$$

and

$$
\gamma_{\mu}^{-}(\varepsilon)=a+\left(2 C_{4} \varepsilon(1+\mu) \Gamma(1+\alpha)\right)^{1 /(1-\alpha)},
$$

and note that $\gamma_{\mu}^{+}(\varepsilon)$ and $\gamma_{\mu}^{-}(\varepsilon)$ are each monotone increasing in $\mu$. Also, let $C_{9}=2 C_{1} C_{3}$.

Lemma 5 Let $\mu>0$ be given. If the Lipschitz constant $\varepsilon$ of $\hat{f}$ is so small that $\gamma_{\mu / C_{9}}^{+}(\varepsilon)>\gamma_{\mu}^{-}(\varepsilon)$ then any semiorbit starting in $K_{\mu}$ remains in $K_{\mu / C_{9}}$ for all positive time.

Proof. Let $\varepsilon>0$ be so small that $\gamma_{\mu / C_{9}}^{+}(\varepsilon)>\gamma_{\mu}^{-}(\varepsilon)$. Let $v(t)+w(t)$ be a semiorbit starting at $v(0)+w(0) \in K_{\mu}$. If $v(0)+w(0)=0$ then the conclusion of the lemma is obvious, so assume that $v(0)+w(0) \neq 0$. Suppose that $v(t)+w(t)$ eventually reaches $\partial K_{\mu / C_{9}}$. Then without loss of generality it may be assumed that $v(0)+w(0) \in \partial K_{\mu}, v(t)+w(t) \in \partial K_{\mu / C_{9}} \backslash\{0\}$, and for $0<\tau<t, v(\tau)+w(\tau) \in K_{\mu / C_{9}} \backslash K_{\mu}$. Using (21) and (22),

$$
\frac{\|v(t)\|_{\alpha}}{\|w(t)\|_{\alpha}} \leq \frac{2 C_{1} C_{3}\|v(0)\|_{\alpha} \exp \left(\gamma_{\mu}^{-}(\varepsilon) t\right)}{\|w(0)\|_{\alpha} \exp \left(\gamma_{\mu / C_{9}}^{+}(\varepsilon) t\right)}=\frac{C_{9}}{\mu} \exp \left(\left(\gamma_{\mu}^{-}(\varepsilon)-\gamma_{\mu / C_{9}}^{+}(\varepsilon)\right) t\right)<\frac{C_{9}}{\mu}
$$

This means $v(t)+w(t) \notin \partial K_{\mu / C_{9}}$, contrary to assumption. This contradiction shows that $v(t)+w(t)$ never reaches $\partial K_{\mu / C_{9}}$, so the positive semiorbit must remain in $K_{\mu / C_{9}}$ for all time.

Let $K_{\mu}^{c}$ represent the closure of the complement of $K_{\mu}$. Restating the preceding lemma in terms of backward semiorbits, yields the following. 
Lemma 6 Let $\mu>0$ be given. If the Lipschitz constant $\varepsilon$ of $\hat{f}$ is so small that $\gamma_{\mu / C_{9}}^{+}(\varepsilon)>\gamma_{\mu}^{-}(\varepsilon)$ then any backward semiorbit starting in $K_{\mu / C_{9}}^{c}$ remains in $K_{\mu}^{c}$ as long as it exists.

The same method that gave the estimates (21) and (22) on the growth and decay of a solution can be used to give similar estimates on the growth and decay of the difference of two solutions. Thus, the cones described above can be centered at points other than the origin, and if the centers are allowed to evolve with the flow, then the corresponding generalizations of Lemmas 5 and 6 hold.

Define the truncated cones

$$
K_{\mu}(r)=\left\{v+w \in K_{\mu}:\|w\|_{\alpha} \leq r\right\}
$$

and

$$
K_{\mu}^{c}(r)=\left\{v+w \in K_{\mu}^{c}:\|v\|_{\alpha} \leq r\right\} .
$$

The following theorem says roughly that a semiorbit starting at a random point near 0 will blow up as $t \rightarrow \infty$, and as it does so it will stay close to $W^{+}$.

Theorem 1 Let $\mu>0$ be given. Suppose the Lipschitz constant $\varepsilon$ of $\hat{f}$ is so small that $W^{+}$exists and is tangent to $X^{+}$at $0, \gamma_{\mu / C_{9}}^{+}(\varepsilon)>0$, and

$$
\gamma_{\mu /\left(2 C_{9}\right)}^{+}(\varepsilon)-\gamma_{C_{9} \mu}^{-}(\varepsilon)>\frac{b-a}{\lambda}
$$

for some $\lambda>1$. Then for any $\delta>0$ and $R>0$ there exists $r>0$ such that any semiorbit $v(t)+w(t)$ starting at $v(0)+w(0) \in K_{\mu}(r) \backslash\{0\}$ will eventually exit the cylinder

$$
\left\{v+w \in X^{\alpha}:\|w\|_{\alpha} \leq R\right\}
$$

and the distance in the $X^{-}$direction from the exit point to $W^{+}$is less than $\delta$. In particular, $r$ will satisfy these conditions if

$$
0<r<\min \left\{\frac{\delta \mu}{8 C_{3}}\left(\frac{\delta \mu}{C_{9}\left(1+C_{9}\right) R}\right)^{\lambda \gamma_{\mu / 2}^{-}(\varepsilon) /(b-a)}, R\right\} .
$$

Proof. Recall that every point on $W^{+}$lies on a backward semiorbit that approaches 0 as $t \rightarrow-\infty$. Since $W^{+}$is tangent to $X^{+}$at 0 , each of these backward semiorbits intersects $K_{C_{9} \mu}$. By the choice of $\varepsilon$,

$$
\gamma_{\mu}^{+}(\varepsilon)>\gamma_{\mu /\left(2 C_{9}\right)}^{+}(\varepsilon)>\gamma_{C_{9} \mu}^{-}(\varepsilon)
$$


so by Lemma 5 it must be true that $W^{+} \subset K_{\mu}$.

Let $u_{2}(0)=v_{2}(0)+w_{2}(0)$ be a point on the base of $K_{\mu / C_{9}}(R)$, and let $u_{1}(0)=v_{1}(0)+w_{1}(0)$ be the point lying on $W^{+}$such that $w_{1}(0)=w_{2}(0)$. Suppose

$$
\left\|v_{1}(0)-v_{2}(0)\right\|_{\alpha} \geq \delta
$$

and suppose $t>0$ is such that the (finite) backward semiorbit

$$
\left\{u_{2}(-\tau):-t \leq-\tau \leq 0\right\}
$$

exists and is contained in $K_{\mu / C_{9}}$. By the choice of $\varepsilon$,

$$
\gamma_{\mu /\left(2 C_{9}\right)}^{+}(\varepsilon)>\gamma_{C_{9} \mu}^{-}(\varepsilon)>\gamma_{\mu / 2}^{-}(\varepsilon)
$$

so the analogue of Lemma 6 for cones centered at $u_{1}(-t)$ says that $u_{2}(-t) \in$ $u_{1}(-t)+K_{\mu / 2}^{c}$. Also, an estimate similar to (22) follows easily:

$$
\delta \leq\left\|v_{2}(0)-v_{1}(0)\right\|_{\alpha} \leq 2 C_{3}\left\|v_{2}(-t)-v_{1}(-t)\right\|_{\alpha} \exp \left(\gamma_{\mu / 2}^{-}(\varepsilon) t\right)
$$

On the other hand,

$$
\frac{\mu}{C_{9}}\left\|v_{2}(-t)\right\|_{\alpha} \leq\left\|w_{2}(-t)\right\|_{\alpha} \leq C_{1} R \exp \left(-\gamma_{\mu / C_{9}}^{+}(\varepsilon) t\right)
$$

By combining (29) and (30) and making use of the triangle inequality and the fact that $u_{1}(-t) \in K_{\mu}$, we get

$$
C_{1} R \exp \left(-\gamma_{\mu / C_{9}}^{+}(\varepsilon) t\right) \geq \frac{\delta \mu}{2 C_{3} C_{9}} \exp \left(-\gamma_{\mu / 2}^{-}(\varepsilon) t\right)-\frac{C_{1} R}{C_{9}} \exp \left(-\gamma_{\mu}^{+}(\varepsilon) t\right)
$$

Thus,

$$
\begin{gathered}
\left(\frac{C_{1} R}{C_{9}}+C_{1} R\right) \exp \left(-\gamma_{\mu / C_{9}}^{+}(\varepsilon) t\right) \geq \frac{\delta \mu}{2 C_{3} C_{9}} \exp \left(-\gamma_{\mu / 2}^{-}(\varepsilon) t\right) \\
\Rightarrow \frac{2 C_{3} C_{9}}{\delta \mu}\left(\frac{C_{1} R}{C_{9}}+C_{1} R\right) \geq \exp \left(\gamma_{\mu / C_{9}}^{+}(\varepsilon) t-\gamma_{\mu / 2}^{-}(\varepsilon) t\right) \\
\Rightarrow \frac{C_{9}\left(1+C_{9}\right) R}{\delta \mu} \geq \exp \left(\frac{b-a}{\lambda} t\right) \\
\Rightarrow t \leq t^{*} \stackrel{\text { def }}{=} \frac{\lambda}{b-a} \ln \left(\frac{C_{9}\left(1+C_{9}\right) R}{\delta \mu}\right)
\end{gathered}
$$

Now suppose, in addition, that $u_{2}(-t) \in K_{\mu}(r)$. Then since $u_{2}(-t) \in$ $u_{1}(-t)+K_{\mu / 2}^{c}$ and $u_{1}(-t) \in K_{\mu}$, it follows from elementary analytic geometry that $u_{1}(-t) \in K_{\mu}(3 r)$. Hence, 


$$
\left\|v_{1}(-t)-v_{2}(-t)\right\|_{\alpha} \leq\left\|v_{1}(-t)\right\|_{\alpha}+\left\|v_{2}(-t)\right\|_{\alpha} \leq \frac{4 r}{\mu}
$$

But

$$
\begin{aligned}
\left\|v_{1}(-t)-v_{2}(-t)\right\|_{\alpha} & \geq \frac{\delta}{2 C_{3}} \exp \left(-\gamma_{\mu / 2}^{-}(\varepsilon) t\right) \\
& \geq \frac{\delta}{2 C_{3}} \exp \left(-\gamma_{\mu / 2}^{-}(\varepsilon) t^{*}\right) \\
& =\frac{\delta}{2 C_{3}}\left(\frac{\delta \mu}{C_{9}\left(1+C_{9}\right) R}\right)^{\lambda \gamma_{\mu / 2}^{-}(\varepsilon) /(b-a)}
\end{aligned}
$$

Combining (31) and (32) gives a contradiction if $r$ satisfies (28). For such $r$, $u_{2}(-t)$ cannot enter $K_{\mu}(r)$ without first exiting $K_{\mu / C_{9}}$.

Now if a (forward) semiorbit starts in $K_{\mu}(r)$ it must exit $K_{\mu / C_{9}}(R)$ through its base, because of the choice of $\varepsilon$. But by the preceding argument, if $r$ satisfies (28) the distance between its exit point and $W^{+}$in the $X^{-}$direction must be less than $\delta$.

Corollary 1 Suppose every nonzero point on the local pseudo-unstable manifold corresponding to (7) has a nontrivial $\omega$-limit set (under ( 7 )) and let $\Omega$ be a neighborhood of the union of these w-limit sets. Let

$$
\Omega^{\prime} \stackrel{\text { def }}{=}\left\{u(0) \in X^{\alpha}: \exists t \geq 0 \text { such that } u(t) \in \Omega\right\},
$$

where $u(t)$ represents the positive semiorbit for (7) beginning at $u(0)$. Then for any $\mu>0$ there exists $r>0$ such that $K_{\mu}(r) \backslash\{0\} \subset \Omega^{\prime}$.

Proof. Let $\varepsilon$ satisfy the hypotheses of Theorem 1 , for some $\lambda>1$. Choose $R$ small enough that $f$ and $\hat{f}$ agree on $K_{\mu / C_{9}}(R)$. Let $S_{R}$ consist of those points $v+w \in X^{+} \oplus X^{-}$that lie on the local pseudo-unstable manifold and satisfy $\|w\|_{\alpha}=R$. Note that any semiorbit beginning on $S_{R}$ enters $\Omega$. Because (7) has continuous dependence on initial data [23], some open subset $V$ of

$$
\left\{v+w \in X^{\alpha}:\|w\|_{\alpha}=R\right\}
$$

containing $S_{R}$ has this same property. Since $X^{+}$is finite-dimensional, $S_{R}$ is compact, so there exists $\delta>0$ such that

$$
\bigcup_{v+w \in S_{R}}\left\{\hat{v}+w \in X^{\alpha}:\|\hat{v}-v\|_{\alpha}<\delta\right\}
$$

is contained in $V$. (See, e.g., [16].) The corollary now follows from an application of Theorem 1 . 
Corollary 2 Let $\Omega$ and $\Omega^{\prime}$ be as in Corollary 1, and let $u_{0} \in X^{\alpha}$ have a nontrivial $X^{+}$component. If $|k|$ is sufficiently small, then $k u_{0} \in \Omega^{\prime}$.

Proof. This is an immediate consequence of Corollary 1.

\subsubsection{Regions Tangent to the Pseudo-Stable Subspace}

Theorem 2 Let $\Omega$ and $\Omega^{\prime}$ be as in Corollary 1. Then there exists $r>0$ and $F: \mathbf{R} \rightarrow \mathbf{R}$ satisfying $F(0)=F^{\prime}(0)=0$ such that if $F\left(\left\|v_{0}\right\|_{\alpha}\right)<\left\|w_{0}\right\|_{\alpha}<r$ then $v_{0}+w_{0} \in \Omega^{\prime}$.

Proof. Apply Corollary 1 with $\mu=1$. Define $F$ as follows: $F$ is even and for $x \geq 0, F(x)$ is the supremum of all $y \leq r$ such that there exists $v+w \in X^{-} \oplus X^{+}$satisfying $\|v\|_{\alpha}=x,\|w\|_{\alpha}=y$, and such that the semiorbit under (7) beginning at $v+w$ never enters $\Omega$. Then from its definition, $F$ has all the desired properties except possibly $F^{\prime}(0)=0$. This property is verified below.

Now the graph of $F$ lies below the graph of $x \mapsto|x|$ on $(-r, r)$. Let $\mu \in(0,1)$. Applying Corollary 1 again produces a secondary truncated cone $K_{\mu}(\hat{r})$. The base of $K_{\mu}(\hat{r})$ hits the lateral surface of $K_{1}(r)$ at some positive distance $d(\mu)$ away from the $X^{+}$axis. Then it is clear that on $(-d(\mu), d(\mu))$ the graph of $F$ lies below the graph of $x \mapsto \mu|x|$. Since this holds for all $\mu \in(0,1)$, it must be true that $F^{\prime}(0)=0$.

\subsubsection{Regions with Boundaries Satisfying a Power Law}

Theorem 2 would be more powerful if it provided more information about the function $F$. A stronger result is possible if a further assumption is made.

Lemma 7 If $\|D f(u)\|_{\mathcal{L}\left(X^{\alpha}, X\right)}=O\left(\|u\|_{\alpha}\right)$ as $u \rightarrow 0$ then the tangency of the pseudo-unstable manifold $W^{+}$at 0 is quadratic. That is, $\|v\|_{\alpha}=O\left(\|w\|_{\alpha}^{2}\right)$ for $u=v+w \in W^{+}$as $u \rightarrow 0$.

Proof. As was mentioned in the proof of Theorem 1, Lemma 5 implies that the pseudo-unstable manifold for (8) is contained in $K_{\mu}$ provided that

$$
\gamma_{\mu}^{+}(\varepsilon)>\gamma_{C_{9} \mu}^{-}(\varepsilon)
$$

Written out explicitly, this condition is

$$
b-C_{2} \varepsilon\left(1+\mu^{-1}\right)>a+\left(2 C_{4} \varepsilon\left(1+C_{9} \mu\right) \Gamma(1+\alpha)\right)^{1 /(1-\alpha)} .
$$

As $\mu \rightarrow \infty$, there exists a constant $k_{1}>0$ such that $\varepsilon$ and $\mu$ will satisfy (33) if 


$$
\varepsilon \leq \frac{k_{1}}{\mu}
$$

Also, Lemma 1 says that there exists a constant $k_{2}>0$ such that $f$ and $\hat{f}$ can be made to agree on $K_{\mu}(R)$ provided that

$$
R \sqrt{1+\mu^{-2}} \leq k_{2} \varepsilon
$$

Thus, for such $R$, the local version of $W^{+}$for (7) (independent of $\varepsilon$ ) exits $K_{\mu}(R)$ through its base, not through its lateral surface. Combining (34) and (35), there is a constant $k_{3}>0$ such that if $R \leq k_{3} / \mu$, then the pseudounstable manifold exits $K_{\mu}(R)$ through its base. This means that if $v+w$ is on this manifold and $\|w\|_{\alpha} \leq k_{3} / \mu$ then $\mu\|v\|_{\alpha} \leq\|w\|_{\alpha}$. In particular, if $\|w\|_{\alpha}=k_{3} / \mu$ then

$$
\|v\|_{\alpha} \leq \frac{1}{\mu}\|w\|_{\alpha}=\frac{1}{k_{3}}\|w\|_{\alpha}^{2}
$$

Hence, $\|v\|_{\alpha}=O\left(\|w\|_{\alpha}^{2}\right)$.

Lemma 8 Let $\Omega$ and $\Omega^{\prime}$ be as in Corollary 1. Suppose that in the proof of Theorem 2, $\delta$ can be chosen so that

$$
\delta \geq \hat{k} R^{q}
$$

as $R \rightarrow 0$ for some $q<2$, and let

$$
E(q) \stackrel{\text { def }}{=} 2-\frac{(2-q) \lambda b}{(2-q) \lambda b+q(b-a)}
$$

Then if $\|D f(u)\|_{\mathcal{L}_{\left(X^{\alpha}, X\right)}}=O\left(\|u\|_{\alpha}\right)$ as $u \rightarrow 0$ there exist $r>0$ and $k>0$ such that if $k\|v\|_{\alpha}^{E(q)}<\|w\|_{\alpha}<r$ then $v+w \in \Omega^{\prime}$.

Proof. Note that in producing the secondary cones in the proof of Theorem 2 , it was sufficient for $\varepsilon$ and $R$ to satisfy

$$
\varepsilon \leq k_{2} \mu
$$

and

$$
R \sqrt{1+C_{9}^{2} \mu^{-2}} \leq k_{3} \varepsilon,
$$

for some constants $k_{2}>0$ and $k_{3}>0$ and for every $\mu$ sufficiently small. Combining (37) and (38) shows that (38) can be replaced by

$$
R \leq k_{4} \mu^{2}
$$


Substituting (36), (37), and (39) into (28) and simplifying gives the estimate

$$
\hat{r} \leq k_{5} \mu^{2+((2-q) \lambda b) /(q(b-a))}
$$

for some constant $k_{5}>0$, as the only requirement on the heights of the secondary cones in the proof of Theorem 2. If $x$ is the radius of one of these secondary cones (in the $X^{-}$direction), then $\mu=\hat{r} / x$. Making this substitution in (40) gives the estimate

$$
\hat{r} \geq k x^{E(q)}
$$

where $E(q)$ is as defined in the statement of the lemma and $k$ is some positive constant depending on $q$. Applying (41) to the proof of Theorem 2 gives the desired result.

The following theorem strengthens the result of Theorem 2 .

Theorem 3 Let $\Omega$ and $\Omega^{\prime}$ be as in Corollary 1. If $\|D f(u)\|_{\mathcal{L}\left(X^{\alpha}, X\right)}=O\left(\|u\|_{\alpha}\right)$ as $u \rightarrow 0$ then for any power $p<\min \{b / a, 2\}$ there exists $r>0$ and $k>0$ such that if $k\|v\|_{\alpha}^{p}<\|w\|_{\alpha}<r$ then $v+w \in \Omega^{\prime}$.

Proof. The theorem is proved by bootstrapping a finite number of times with Lemma 8. To start off, apply Corollary 1 with $\mu=1$, to get a finite cone contained in $\Omega^{\prime}$. Using this cone and Lemma 7 it can be seen that for $R$ sufficiently small the $\delta$ in Theorem 2 can be taken of the same order as $R$. That is, there is a constant $k_{1}>0$ such that, for $R$ small, if

$$
\delta \leq k_{1} R
$$

then every point on the cylinder

$$
\left\{v+w \in X^{\alpha}:\|w\|_{\alpha}=R\right\}
$$

whose distance from $W^{+}$in the $X^{-}$direction is no bigger than $\delta$ lies in $\Omega^{\prime}$. Thus, Lemma 8 can be applied with $q=1$. Just as the existence of the cone in $\Omega^{\prime}$ allowed the application of this lemma with $q=1$, the conclusion of this lemma with $q=1$ allows us to apply the lemma again with $q=E(1)$. This procedure can be iterated as many times as desired, to get better and better power laws for a subset of $\Omega^{\prime}$. A straightforward calculation shows that $E$ is a strictly increasing function of $q$ if

$$
q<\min \left\{\frac{\lambda b}{(\lambda-1) b+a}, 2\right\} .
$$


Hence, either

$$
E^{n}(1) \rightarrow \min \left\{\frac{\lambda b}{(\lambda-1) b+a}, 2\right\}
$$

as $n \rightarrow \infty$ or

$$
E^{n}(1) \geq \min \left\{\frac{\lambda b}{(\lambda-1) b+a}, 2\right\}
$$

for some $n \in \mathbf{N}$. By taking $\lambda$ sufficiently close to 1 , we obtain the desired result.

\subsubsection{Measure-theoretic Results}

Theorems 2 and 3 each identify a region of initial data near the origin whose positive semiorbits are, in some sense, controlled by the pseudo-unstable manifold. It is natural to try to find some way of determining the significance of this region, and a natural way of doing this is finding its size with respect to some measure. A very general measure-theoretic restatement of the results of the previous sections is presented in a theorem below. The following definition will be helpful: A subset $S$ of a topological vector space is balanced if, for any scalar $\alpha$ satisfying $|\alpha| \leq 1, \alpha S \subset S$.

Lemma 9 If $S$ is balanced then $\lambda S$ is balanced for any scalar $\lambda$. Also, the union of balanced sets is balanced.

Proof. Verification of the lemma is straightforward.

Theorem 4 Let $\Omega$ and $\Omega^{\prime}$ be as in Corollary 1, and let $\varphi$ be a probability measure on the Borel sets of $X^{\alpha}$. Given $\varepsilon>0$ let $\varphi_{\varepsilon}$ be the scaled probability measure defined by $\varphi_{\varepsilon}(V)=\varphi\left(\varepsilon^{-1} V\right)$. Suppose $\varphi\left(X^{-} \cap X^{\alpha}\right)=0$. Then $\varphi_{\varepsilon}\left(\Omega^{\prime}\right) \rightarrow 1$ as $\varepsilon \rightarrow 0$.

Proof. Define $S$ to be the union of the (closed) finite cones constructed in the proof of Theorem 2. Since each cone is balanced, by the second part of Lemma 9, $S$ is also balanced. Applying the first part of Lemma $9, \varepsilon^{-1} S$ is balanced for any $\varepsilon>0$. Hence, $\varepsilon_{i}^{-1} S \subset \varepsilon_{j}^{-1} S$ if $\varepsilon_{i}>\varepsilon_{j}$.

Now, let $\left(\varepsilon_{k}\right)$ be a sequence of positive real numbers converging to 0 . By Corollary 2,

$$
X^{\alpha} \backslash X^{-} \subset \bigcup_{k=1}^{\infty} \frac{1}{\varepsilon_{k}}(S \backslash\{0\}),
$$

SO

$$
\begin{aligned}
1 & =\varphi\left(X^{\alpha}\right) \\
& =\varphi\left(X^{\alpha} \backslash X^{-}\right)+\varphi\left(X^{\alpha} \cap X^{-}\right)
\end{aligned}
$$




$$
\begin{aligned}
& =\varphi\left(X^{\alpha} \backslash X^{-}\right) \\
& \leq \varphi\left(\bigcup_{k=1}^{\infty} \frac{1}{\varepsilon_{k}}(S \backslash\{0\})\right) \\
& =\varphi\left(\bigcup_{k=1}^{\infty} \frac{1}{\varepsilon_{k}} S\right) \\
& =\lim _{k \rightarrow \infty} \varphi\left(\frac{1}{\varepsilon_{k}} S\right) \\
& =\lim _{k \rightarrow \infty} \varphi\left(\frac{1}{\varepsilon_{k}}(S \backslash\{0\})\right) \\
& \leq \lim _{k \rightarrow \infty} \varphi\left(\frac{1}{\varepsilon_{k}} \Omega^{\prime}\right) \\
& =\lim _{k \rightarrow \infty} \varphi_{\varepsilon_{k}}\left(\Omega^{\prime}\right) \\
& \leq 1 .
\end{aligned}
$$

This proves the theorem.

Let us now turn our attention to a particular class of probability measures for which the preceding theorem can be strengthened. Probably the most important measure on $\mathbf{R}$ that is concentrated near one particular point is one-dimensional Gaussian measure $\varphi$ defined by

$$
\varphi(E)=\frac{1}{\sqrt{2 \pi \sigma^{2}}} \int_{E} \exp \left(-\frac{(x-m)^{2}}{2 \sigma^{2}}\right) d x .
$$

This measure corresponds to the normal distribution with mean $m$ and variance $\sigma^{2}$. A point mass measure may be considered to be a degenerate Gaussian measure with variance 0 . A Borel measure $\varphi$ on a Hilbert space $H$ is said to be a Gaussian measure if each of its projections onto one-dimensional subspaces gives a one-dimensional Gaussian measure; i.e., $\langle\cdot, h\rangle$ is normally-distributed for each $h \in H$. More precisely, if $\mathcal{S}\{h\}$ is the span of $h \in H$, the measure $\varphi_{h}$ on $\mathbf{R}$ given by

$$
\varphi_{h}(E)=\varphi\left(E h \times(\mathcal{S}\{h\})^{\perp}\right)
$$

must be a one-dimensional Gaussian measure. Such measures are easiest to deal with when $H$ is separable, so assume that this is true for the remainder of this section. Also, for simplicity only Gaussian measures with mean 0 (i.e., each projection has mean 0) will be considered. Nontrivial Gaussian measures are easy to construct on separable Hilbert spaces by specifying the projections onto an orthonormal system of vectors. These projections cannot, however, be arbitrarily chosen; the variance of the $n$th projection must approach 0 at a certain rate as $n \rightarrow \infty$. See Skorohod [22] for details. 
The following lemma shows how a Gaussian measure behaves with respect to subspaces.

Lemma 10 Let $H$ be a separable Hilbert space and $\varphi$ a Gaussian measure on $H$ with mean 0 . Let $A \subset H$ be a Hilbert space, and define $\varphi^{A}$ to be the measure on A satisfying

$$
\varphi^{A}(U)=\varphi\left(U \times A^{\perp}\right)
$$

for every $U \subset A$. Then $\varphi^{A}$ is a Gaussian measure with mean 0 .

Proof. Let $a \in A$, and let $E \subset \mathbf{R}$. Let $A^{\prime}$ be the orthogonal complement of $\mathcal{S}\{a\}$ in $A$. Then

$$
\begin{aligned}
\varphi_{a}^{A}(E) & =\varphi^{A}\left(E a \times A^{\prime}\right) \\
& =\varphi\left(\left(E a \times A^{\prime}\right) \times A^{\perp}\right) \\
& =\varphi\left(E a \times\left(A^{\prime} \times A^{\perp}\right)\right) \\
& =\varphi\left(E a \times(\mathcal{S}\{a\})^{\perp}\right),
\end{aligned}
$$

where $(\mathcal{S}\{a\})^{\perp}$ is the orthogonal complement of $\mathcal{S}\{a\}$ in $H$. Since $\varphi$ is a Gaussian measure with mean 0, (42) implies that $\varphi_{a}^{A}$ is a one-dimensional Gaussian measure. Since this holds for any $a \in A, \varphi^{A}$ must be a Gaussian measure with mean 0 .

The covariance operator $S_{\varphi}$ of a measure $\varphi$ on a Hilbert space $H$ is the bounded linear operator on $H$ defined by

$$
\left\langle S_{\varphi} x, y\right\rangle=\int_{H}\langle x, z\rangle\langle y, z\rangle d \varphi(z)
$$

For an arbitrary measure the covariance operator may not exist; however, it is clear that if $S_{\varphi}$ exists it must be positive semidefinite and self-adjoint. Furthermore, a result due to Prohorov found in Kuo [13] shows that if $\varphi$ is a Gaussian measure on a separable Hilbert space then $S_{\varphi}$ exists and its trace is finite. This fact allows one to estimate certain integrals with respect to $\varphi$. The following result, which estimates how fast Gaussian measures must die out at infinity, makes use of some work by Kuo [13].

Lemma 11 Let $\varphi$ be a Gaussian measure with mean 0 on the separable Hilbert space $H$. Then there exist positive constants $M$ and $k$ such that for any $R \geq 0$

$$
\varphi(\{h \in H:\|h\| \geq R\}) \leq M e^{-k R^{2}}
$$


Proof. Since $S_{\varphi}$ is bounded, positive semidefinite, and self-adjoint, $H$ has an orthonormal basis given by the eigenvectors $\left\{e_{n}\right\}$ of $S_{\varphi}$, with corresponding (nonnegative) eigenvalues $\left\{\alpha_{n}\right\}$. Note that

$$
\int_{H}\left\langle x, e_{n}\right\rangle^{2} d \varphi(x)=\left\langle S_{\varphi} e_{n}, e_{n}\right\rangle=\left\langle\alpha_{n} e_{n}, e_{n}\right\rangle=\alpha_{n},
$$

so $\left\langle\cdot, e_{n}\right\rangle$ is normally distributed with mean 0 and variance $\alpha_{n}$. Choose

$$
k \in\left(0, \inf \left\{\frac{1}{2 \alpha_{n}}: n \in \mathbf{N}, \alpha_{n} \neq 0\right\}\right) .
$$

This choice is possible since the boundedness of $S_{\varphi}$ implies that the $\alpha_{n}$ are bounded above. Now

$$
\begin{aligned}
\int_{H} e^{k\|x\|^{2}} d \varphi(x) & =\int_{H} e^{k \sum_{n}\left\langle x, e_{n}\right\rangle^{2}} d \varphi(x) \\
& =\int_{H} \prod_{n} e^{k\left\langle x, e_{n}\right\rangle^{2}} d \varphi(x) \\
& =\prod_{n} \int_{H} e^{k\left\langle x, e_{n}\right\rangle^{2}} d \varphi(x) .
\end{aligned}
$$

A routine computation gives

$$
\int_{H} e^{k\left\langle x, e_{n}\right\rangle^{2}} d \varphi(x)=\int_{0}^{\infty} \varphi\left(\left\{x \in H: e^{k\left\langle x, e_{n}\right\rangle^{2}} \geq y\right\}\right) d y=\left(1-2 k \alpha_{n}\right)^{-1 / 2}
$$

so (43) becomes

$$
\begin{aligned}
\int_{H} e^{k\|x\|^{2}} d \varphi(x) & =\prod_{n}\left(1-2 k \alpha_{n}\right)^{-1 / 2} \\
& =\left(\prod_{n}\left(1-2 k \alpha_{n}\right)\right)^{-1 / 2}
\end{aligned}
$$

Since

$$
\sum_{n} 2 k \alpha_{n}=2 k \sum_{n} \alpha_{n}=2 k \cdot \operatorname{tr}\left(S_{\varphi}\right)<\infty,
$$

the infinite product in (44) converges to a positive number. (See, e.g., Rudin [21].)

Define

$$
M=\int_{H} e^{k\|x\|^{2}} d \varphi(x)
$$

and

$$
H_{R}=\{h \in H:\|h\| \geq R\} .
$$

Then from (44), 


$$
\begin{aligned}
\varphi\left(H_{R}\right) & =\int_{H_{R}} 1 d \varphi(x) \\
& \leq \int_{H_{R}} e^{k\|x\|^{2}-k R^{2}} d \varphi(x) \\
& =e^{-k R^{2}} \int_{H_{R}} e^{k\|x\|^{2}} d \varphi(x) \\
& \leq e^{-k R^{2}} \int_{H} e^{k\|x\|^{2}} d \varphi(x) \\
& =M e^{-k R^{2}} .
\end{aligned}
$$

This completes the proof.

These results permit an estimate on the rate of convergence in Theorem 4 when $\varphi$ is Gaussian.

Theorem 5 Let $\Omega$ and $\Omega^{\prime}$ be as in Corollary 1, and assume that

$$
\|D f(u)\|_{\mathcal{L}\left(X^{\alpha}, X\right)}=O\left(\|u\|_{\alpha}\right)
$$

and $X^{\alpha}$ is separable. Let $\varphi$ be a Gaussian measure on $X^{\alpha}$ with mean 0 such that for all $x \in X^{+}$the induced variance of $\langle\cdot, x\rangle$ is greater than 0 . If $\varphi_{\varepsilon}$ is defined as in Theorem 4, then

$$
\varphi_{\varepsilon}\left(\Omega^{\prime}\right)=1-O\left(\varepsilon^{n(p-1)}\right)
$$

for any $p<\min \{b / a, 2\}$, where $n=\operatorname{dim}\left(X^{+}\right)$.

Proof. Choose $r>0, k>0$, and

$$
p^{\prime} \in\left(p, \min \left\{\frac{b}{a}, 2\right\}\right)
$$

such that

$$
S \stackrel{\text { def }}{=}\left\{v+w \in X^{\alpha}: k\|v\|_{\alpha}^{p^{\prime}}<\|w\|_{\alpha}<r\right\}
$$

is contained in $\Omega^{\prime}$. This is possible because of Theorem 3. Note that for $\varepsilon>0$ sufficiently small, $S$ contains the set

$$
\bar{S} \stackrel{\text { def }}{=}\left\{v+w \in X^{\alpha}: \varepsilon^{p}<\|w\|_{\alpha}<r,\|v\|_{\alpha}<\left(\frac{\varepsilon^{p}}{k}\right)^{1 / p^{\prime}}\right\} .
$$

Thus,

$$
\begin{aligned}
\varphi_{\varepsilon}\left(\Omega^{\prime}\right) & \geq \varphi_{\varepsilon}(S) \\
& \geq \varphi_{\varepsilon}(\bar{S})
\end{aligned}
$$




$$
\begin{aligned}
= & \varphi\left(\frac{1}{\varepsilon} \bar{S}\right) \\
\geq \quad & -\varphi\left(\left\{v+w \in X^{\alpha}:\|w\|_{\alpha} \leq \varepsilon^{p-1}\right\}\right) \\
& -\varphi\left(\left\{v+w \in X^{\alpha}:\|w\|_{\alpha} \geq \frac{r}{\varepsilon}\right\}\right) \\
& -\varphi\left(\left\{v+w \in X^{\alpha}:\|v\|_{\alpha} \geq \frac{1}{\varepsilon}\left(\frac{\varepsilon^{p}}{k}\right)^{1 / p^{\prime}}\right\}\right) \\
= & 1-\varphi^{X^{+}}\left(\left\{w \in X^{+}:\|w\|_{\alpha} \leq \varepsilon^{p-1}\right\}\right) \\
& -\varphi^{X^{+}}\left(\left\{w \in X^{+}:\|w\|_{\alpha} \geq \frac{r}{\varepsilon}\right\}\right) \\
& -\varphi^{X^{-} \cap X^{\alpha}}\left(\left\{v \in X^{-} \cap X^{\alpha}:\|v\|_{\alpha} \geq \frac{1}{\varepsilon}\left(\frac{\varepsilon^{p}}{k}\right)^{1 / p^{\prime}}\right\}\right) .
\end{aligned}
$$

Now for some constant $C_{1}>0$

$$
\varphi^{X^{+}}\left(\left\{w \in X^{+}:\|w\|_{\alpha} \leq \varepsilon^{p-1}\right\}\right) \leq C_{1}\left(\varepsilon^{p-1}\right)^{n}
$$

where $n=\operatorname{dim}\left(X^{+}\right)$. This estimate comes from the simple observation that an $n$-dimensional Gaussian measure for which no projection is a point mass is absolutely continuous with respect to $n$-dimensional Lebesgue measure. By Lemmas 10 and 11, the following estimates obtain:

$$
\begin{gathered}
\varphi^{X^{+}}\left(\left\{w \in X^{+}:\|w\|_{\alpha} \geq \frac{r}{\varepsilon}\right\}\right) \leq C_{2} \exp \left[-k_{2}\left(\frac{r}{\varepsilon}\right)^{2}\right] \\
\varphi^{X^{-} \cap X^{\alpha}}\left(\left\{v \in X^{-} \cap X^{\alpha}:\|v\|_{\alpha} \geq \frac{1}{\varepsilon}\left(\frac{\varepsilon^{p}}{k}\right)^{1 / p^{\prime}}\right\}\right) \\
\leq C_{3} \exp \left[-k_{3}\left(\frac{1}{\varepsilon}\left(\frac{\varepsilon^{p}}{k}\right)^{1 / p^{\prime}}\right)^{2}\right] \\
\leq C_{3} \exp \left[-k_{4} \varepsilon^{2\left(p / p^{\prime}-1\right)}\right] .
\end{gathered}
$$

Estimates (47) and (48) say that the corresponding quantities in (45) are transcendentally small as $\varepsilon \rightarrow 0$. Therefore, substituting (46) into (45) gives

$$
\varphi_{\varepsilon}\left(\Omega^{\prime}\right) \geq 1-O\left(\varepsilon^{n(p-1)}\right)
$$

Since $\varphi_{\varepsilon}\left(\Omega^{\prime}\right) \leq 1$, the desired result follows immediately. 


\section{Spinodal Decomposition}

\subsection{The Equation in an Abstract Setting}

We now return to the Cahn-Hilliard equation. Let $M$ lie in the spinodal region of $W$ so that $W^{\prime \prime}(M)<0$. Recall that the constant $u \equiv M$ is an equilibrium for the Cahn-Hilliard equation. The goal in this section is to show that the results of Section 3 regarding the nature of a semiflow in a neighborhood of an equilibrium can be be applied to the Cahn-Hilliard equation, by showing that the Cahn-Hilliard equation fits into the abstract setting described in Section 3.1 .

The first thing to do is to make the change of variable $\hat{u}=u-M$ so that the particular equilibrium of interest ends up at the origin. Simultaneously, let $\beta^{2}=-W^{\prime \prime}(M)>0$ (as in Section 2) and define the function $\psi$ by

$$
\psi(\hat{u})=W^{\prime}(\hat{u}+M)+\beta^{2} \hat{u} .
$$

(Since $W$ is $C^{5}, \psi$ is $C^{4}$.) Making these substitutions into (1) and dropping the caret from $\hat{u}$ gives

$$
\begin{array}{rl}
\frac{\partial u}{\partial t}=-\Delta\left(\varepsilon^{2} \Delta u+\beta^{2} u-\psi(u)\right) & x \in \Omega \\
\frac{\partial u}{\partial \nu}=\frac{\partial \Delta u}{\partial \nu}=0 & x \in \partial \Omega .
\end{array}
$$

Define

$$
A u=-\Delta\left(\varepsilon^{2} \Delta u+\beta^{2} u\right),
$$

and let

$$
f(u)=\Delta(\psi(u)),
$$

so $(50)$ is of the form

$$
u_{t}=A u+f(u)
$$

with $f(0)=0$ as required. Let

$$
X=\left\{u \in L^{2}(\Omega): \int_{\Omega} u d x=0\right\}
$$

and

$$
\mathcal{D}(A)=\left\{u \in X \cap H^{4}(\Omega):\left.\frac{\partial u}{\partial \nu}\right|_{\partial \Omega}=\left.\frac{\partial \Delta u}{\partial \nu}\right|_{\partial \Omega}=0\right\} .
$$

Because the Cahn-Hilliard equation conserves mass, spaces with integral constraints are the most appropriate spaces with which to work. By Rankin [20], 
the linear operator $-A$ with the given domain is a sectorial operator, so $A$ generates an analytic semigroup $S(t)$. Also,

$$
X^{1 / 2}=\left\{u \in X \cap H^{2}(\Omega):\left.\frac{\partial u}{\partial \nu}\right|_{\partial \Omega}=0\right\} .
$$

Note that when $\Omega=[0,1]$, an explicit spectral representation for $S(t)$ was derived in the process of performing the linear analysis.

In order to be able to use the results of Section 3 with $\alpha=1 / 2$, it is necessary to show that $f$ is continuously differentiable from $X^{1 / 2}$ to $X$ with $D f(0)=0$; if such results as Theorems 3 and 5 are to be used then it must also be verified that

$$
\|D f(u)\|_{\mathcal{L}\left(X^{1 / 2}, X\right)}=O\left(\|u\|_{X^{1 / 2}}\right)
$$

These verifications will be performed here under the assumption that $\operatorname{dim}(\Omega) \leq$ 3. Expanding $f(u)$ gives

$$
f(u)=\Delta(\psi(u))=\psi^{\prime}(u) \Delta u+\psi^{\prime \prime}(u)|\nabla u|^{2} .
$$

The Sobolev imbedding theorems (see, e.g., Gilbarg and Trudinger [8]) imply that $H^{2}(\Omega) \hookrightarrow L^{\infty}(\Omega) \cap W^{1,4}(\Omega)$; therefore, if $u \in H^{2}(\Omega)$ then $f(u) \in L^{2}(\Omega)$. Also, if $u \in X^{1 / 2}$ then $\frac{\partial u}{\partial \nu}=0$ on $\partial \Omega$, so by the divergence theorem

$$
\int_{\Omega} f(u) d x=\int_{\Omega} \Delta(\psi(u)) d x=\int_{\partial \Omega} \frac{\partial \psi(u)}{\partial \nu} d x=0 .
$$

Since $f(u) \in L^{2}(\Omega)$ and has mean value $0, f(u) \in X$. Hence, $f: X^{1 / 2} \rightarrow X$, as was needed.

Next consider the linear mapping $h \mapsto \Delta\left(\psi^{\prime}(u) h\right)$ as a candidate for $D f(u)$. By expanding and repeatedly applying the triangle inequality, we get the estimate

$$
\begin{aligned}
\| \Delta(\psi & (u+h))-\Delta(\psi(u))-\Delta\left(\psi^{\prime}(u) h\right) \|_{L^{2}} \\
\leq \quad & \left\|\left(\psi^{\prime}(u+h)-\psi^{\prime}(u)\right) \Delta h\right\|_{L^{2}} \\
& +2\left\|\left(\psi^{\prime \prime}(u+h)-\psi^{\prime \prime}(u)\right) \nabla u \cdot \nabla h\right\|_{L^{2}} \\
& +\left\|\left(\psi^{\prime}(u+h)-\psi^{\prime}(u)\right) \Delta u-\psi^{\prime \prime}(u) h \Delta u\right\|_{L^{2}} \\
& +\left\|\left(\psi^{\prime \prime}(u+h)-\psi^{\prime \prime}(u)\right)|\nabla u|^{2}-\psi^{\prime \prime \prime}(u)|\nabla u|^{2} h\right\|_{L^{2}} \\
& +\left\|\psi^{\prime \prime}(u+h)|\nabla h|^{2}\right\|_{L^{2}} \\
\stackrel{\text { def }}{=} & N_{1}+2 N_{2}+N_{3}+N_{4}+N_{5} .
\end{aligned}
$$


Applying the mean value theorem to $N_{1}$ and $N_{2}$ and to $N_{3}$ and $N_{4}$ twice gives

$$
\begin{aligned}
& N_{1} \leq\left\|\psi^{\prime \prime}\left(\theta_{1}\right) h \Delta h\right\|_{L^{2}} \\
& N_{2} \leq\left\|\psi^{\prime \prime \prime}\left(\theta_{2}\right) h \nabla u \cdot \nabla h\right\|_{L^{2}} \\
& N_{3} \leq\left\|\psi^{\prime \prime \prime}\left(\theta_{3}\right) h^{2} \Delta u\right\|_{L^{2}} \\
& N_{4} \leq\left\|\psi^{\prime \prime \prime \prime}\left(\theta_{4}\right) h^{2}|\nabla u|^{2}\right\|_{L^{2}}
\end{aligned}
$$

where the $\theta_{j}$ are functions that are pointwise between $u$ and $u+h$. Applying Hölder's inequality yields

$$
\begin{aligned}
& N_{1} \leq\left\|\psi^{\prime \prime}\left(\theta_{1}\right)\right\|_{L^{\infty}}\|h\|_{L^{\infty}}\|\Delta h\|_{L^{2}} \\
& N_{2} \leq\left\|\psi^{\prime \prime \prime}\left(\theta_{2}\right)\right\|_{L^{\infty}}\|h\|_{L^{\infty}}\|\nabla u\|_{L^{4}}\|\nabla h\|_{L^{4}} \\
& N_{3} \leq\left\|\psi^{\prime \prime \prime}\left(\theta_{3}\right)\right\|_{L^{\infty}}\|h\|_{L^{\infty}}^{2}\|\Delta u\|_{L^{2}} \\
& N_{4} \leq\left\|\psi^{\prime \prime \prime \prime}\left(\theta_{4}\right)\right\|_{L^{\infty}}\|h\|_{L^{\infty}}^{2}\|\nabla u\|_{L^{4}}^{2} \\
& N_{5} \leq\left\|\psi^{\prime \prime}(u+h)\right\|_{L^{\infty}}\|\nabla h\|_{L^{4}}^{2},
\end{aligned}
$$

so by the Sobolev imbedding theorems, (51) gives

$$
\left\|\Delta(\psi(u+h))-\Delta(\psi(u))-\Delta\left(\psi^{\prime}(u) h\right)\right\|_{L^{2}}=O\left(\|h\|_{H^{2}}^{2}\right)
$$

as $\|h\|_{H^{2}} \rightarrow 0$. Also,

$$
\begin{aligned}
\left\|\Delta\left(\psi^{\prime}(u) h\right)\right\|_{L^{2}} \leq & \left\|\psi^{\prime \prime \prime}(u)|\nabla u|^{2} h\right\|_{L^{2}} \\
& +\left\|\psi^{\prime \prime}(u) h \Delta u\right\|_{L^{2}} \\
& +2\left\|\psi^{\prime \prime}(u) \nabla u \cdot \nabla h\right\|_{L^{2}} \\
& +\left\|\psi^{\prime}(u) \Delta h\right\|_{L^{2}} \\
\leq & \left\|\psi^{\prime \prime \prime}(u)\right\|_{L^{\infty}}\|\nabla u\|_{L^{4}}^{2}\|h\|_{L^{\infty}} \\
& +\left\|\psi^{\prime \prime}(u)\right\|_{L^{\infty}}\|\Delta u\|_{L^{2}}\|h\|_{L^{\infty}} \\
& +2\left\|\psi^{\prime \prime}(u)\right\|_{L^{\infty}}\|\nabla u\|_{L^{4}}\|\nabla h\|_{L^{4}} \\
& +\left\|\psi^{\prime}(u)\right\|_{L^{\infty}}\|\Delta h\|_{L^{2}} .
\end{aligned}
$$

By $(49), \psi^{\prime}(0)=0$, so

$$
\left\|\psi^{\prime}(u)\right\|_{L^{\infty}}=O\left(\|u\|_{L^{\infty}}\right)
$$

hence,

$$
\left\|\Delta\left(\psi^{\prime}(u) h\right)\right\|_{L^{2}}=O\left(\|u\|_{H^{2}}\|h\|_{H^{2}}\right) .
$$

The combination of (52) and (53) implies that $f: H^{2} \rightarrow L^{2}$ is differentiable, $D f(u) h=\Delta\left(\psi^{\prime}(u) h\right)$, and 


$$
\|D f(u)\|_{\mathcal{L}\left(H^{2}, L^{2}\right)}=O\left(\|u\|_{H^{2}}\right)
$$

In particular, this means that $D f(0)=0$. The verification of the fact that the map $u \mapsto D f(u)$ is continuous from $H^{2}$ to $\mathcal{L}\left(H^{2}, L^{2}\right)$ is essentially the same as the derivation of (53), so the details will be omitted.

Now recall that all of the preceding estimates held for $\Omega \subset \mathbf{R}^{n}, n \leq 3$, so the results of Section 3 can be applied to the Cahn-Hilliard equation on any such $\Omega$, provided that an appropriate decomposition $X^{-} \oplus X^{+}$of $X$ is chosen. However, for these results to be very illuminating, something must be known about the $\omega$-limit set of an arbitrary point on the pseudo-unstable manifold of interest. Such information is difficult to obtain in spaces of high dimension, so for the rest of this section consider $\Omega=[0,1] \subset \mathbf{R}$. As was mentioned in Section 2 , generically there is a single fastest-growing mode, say $\cos n_{0} \pi x$, for

the linearization of the Cahn-Hilliard equation about $u \equiv 0$. Suppose $\varepsilon>0$ is such that this generic phenomenon is realized, and let $X^{+}$be the span of $\cos n_{0} \pi x$ and $X^{-}$be the orthogonal subspace consisting of all other modes. This decomposition satisfies all of the conditions in Section 3.

\subsection{Properties of the $\omega$-limit Points}

Now return to the original coordinate system where the equilibrium of interest lies at $u \equiv M$. Because the Cahn-Hilliard equation has a Lyapunov functional, namely van der Waal's free energy functional, and because this functional is strictly decreasing along nonequilibrium trajectories, the $\omega$-limit set of any point consists entirely of equilibria. (The dissipativity of the CahnHilliard equation implies that each $\omega$-limit set is nonempty.) By integrating (3) and using the corresponding boundary conditions, it can be seen that an equilibrium $u(x)$ of the one-dimensional Cahn-Hilliard equation must satisfy

$$
-\varepsilon^{2} u^{\prime \prime}(x)+W^{\prime}(u(x))=c
$$

for some constant $c$ and boundary conditions

$$
u^{\prime}(0)=u^{\prime}(1)=0
$$

Since the only equilibria of interest are those with the same mass as the initial data, $c$ is not arbitrary but must be such that $\int_{0}^{1} u(x) d x=M$. By defining $v(x)=u^{\prime}(x)$ and observing the symmetry of the phase plane of the $(u, v)$ system about the $u$-axis, it is easy to see that $u$ must either be strictly monotone (in which case it can be thought of as being periodic with period 2) or 
$2 / k$-periodic for some positive integer $k$. Also, each period is made up of two reflection-symmetric monotone transition layers of width $1 / k$.

Now consider the pseudo-unstable manifold tangent to the $X^{+}$axis, where $X^{+}$is as defined above. Consider the closed subspace $Y \subset X$ consisting of elements whose Fourier expansions contain only $\cos n_{0} \pi x$ and its higher harmonics, i.e, $\cos 2 n_{0} \pi x, \cos 3 n_{0} \pi x$, etc. This space is clearly invariant under (3), so the methods of Section 3 could be applied to $Y$ instead of $X$ to obtain a pseudo-unstable manifold tangent to $X^{+}$and contained within $Y$. Because of the characterization of pseudo-unstable manifolds in terms of asymptotic growth rates, they are unique, so these two manifolds must be the same; consequently, the original pseudo-unstable manifold is contained in $Y$. Since $Y$ is closed, it also contains the $\omega$-limit sets of every nonzero point on the pseudo-unstable manifold. Hence, any equilibrium found in such an $\omega$-limit set must have minimal period equal to $2 /\left(k n_{0}\right)$ (and, therefore, transition layers of width $\left.1 /\left(k n_{0}\right)\right)$ for some positive integer $k$. In particular, this transition length is $O(\varepsilon)$ as $\varepsilon \rightarrow 0$.

It is not too hard to relate the periodicity of a steady-state solution for the Cahn-Hilliard equation to its amplitude. The following proposition, which is a special case of the Sturm comparison theorem [10], will be useful in this regard.

Proposition 1 Let $v_{1}$ and $v_{2}$ satisfy

$$
\begin{aligned}
& -\varepsilon^{2} v_{1}^{\prime \prime}(x)+a_{1}(x) v_{1}(x)=0 \\
& -\varepsilon^{2} v_{2}^{\prime \prime}(x)+a_{2}(x) v_{2}(x)=0
\end{aligned}
$$

on $[0,1]$ with initial conditions

$$
\begin{aligned}
& v_{1}(0)=v_{2}(0)=0 \\
& v_{1}^{\prime}(0)=v_{2}^{\prime}(0) \neq 0 .
\end{aligned}
$$

Suppose $a_{1}(x) \geq a_{2}(x)$ for all $x \in[0,1]$. Then if $v_{1}\left(x_{1}\right)=0$ for some $x_{1} \in(0,1]$ then $v_{2}\left(x_{2}\right)=0$ for some $x_{2} \in\left(0, x_{1}\right]$.

Now given a fixed equilibrium $u(x)$ that is an $\omega$-limit point of a point on the pseudo-unstable manifold, let $v(x)=u^{\prime}(x)$. Clearly $v$ satisfies

$$
-\varepsilon^{2} v^{\prime \prime}(x)+W^{\prime \prime}(u(x)) v(x)=0
$$

with boundary conditions

$$
v(0)=v(1)=0 .
$$


Since energy decreases along trajectories of the Cahn-Hilliard equation, it is impossible for $u$ to be the homogeneous equilibrium $u \equiv M$. This means that $v^{\prime}(0) \neq 0$ since, otherwise, the uniqueness of solutions to initial value problems would imply that $v \equiv 0$. Hence, Proposition 1 can be applied with either $v=v_{1}$ or $v=v_{2}$ if a suitable complementary comparison function can be found. Let $a^{+}=\sup _{s \in[0,1]} W^{\prime \prime}(u(s))$, and let $a^{-}=\inf _{s \in[0,1]} W^{\prime \prime}(u(s))$. Applying Proposition 1 with $a_{1}(x) \equiv a^{+}, a_{2}(x) \equiv W^{\prime \prime}(u(x))$, and $v_{1}^{\prime}(0)=v_{2}^{\prime}(0)=v(0)$ gives the estimate that the first positive zero of $v_{2}(x) \equiv v(x)$ comes no later than the first positive zero of

$$
v_{1}(x) \equiv \frac{\varepsilon v^{\prime}(0)}{\sqrt{-a^{+}}} \sin \left(\frac{\sqrt{-a^{+}}}{\varepsilon} x\right)
$$

if $a^{+}<0$. This means that the transition width $1 /\left(k n_{0}\right)$ of $u$ is less than or equal to

$$
\frac{\pi \varepsilon}{\sqrt{-a^{+}}}
$$

if $a^{+}<0$. Written another way,

$$
-\pi^{2} \varepsilon^{2} k^{2} n_{0}^{2} \leq a^{+}
$$

and this clearly holds if $a^{+} \geq 0$, as well. On the other hand, applying Proposition 1 with $a_{1}(x) \equiv W^{\prime \prime}(u(x)), a_{2}(x) \equiv a^{-}$, and the same initial conditions as before gives the estimate

$$
a^{-} \leq-\pi^{2} \varepsilon^{2} k^{2} n_{0}^{2}
$$

Since (56) and (57) estimate the range of $W^{\prime \prime} \circ u$, they give bounds on the amplitude of $u$. To see whether these bounds are meaningful, it helps to recall the value of $n_{0}$. In Section 3.1 it was shown that

$$
\left|n_{0}-\frac{\beta}{\varepsilon \pi \sqrt{2}}\right|<1
$$

SO

$$
n_{0}=\frac{\beta}{\varepsilon \pi \sqrt{2}}+O(1)
$$

as $\varepsilon \rightarrow 0$. Therefore,

$$
-\pi^{2} \varepsilon^{2} k^{2} n_{0}^{2}=-\frac{\beta^{2} k^{2}}{2}+O(\varepsilon)=\frac{k^{2} W^{\prime \prime}(M)}{2}+O(\varepsilon) .
$$

Substituting this into (56) and (57) and using the definition of $a^{ \pm}$, we have 


$$
\inf _{s \in[0,1]} W^{\prime \prime}(u(s)) \leq \frac{k^{2} W^{\prime \prime}(M)}{2}+O(\varepsilon) \leq \sup _{s \in[0,1]} W^{\prime \prime}(u(s)) .
$$

Now since the average value of $u$ is $M$,

$$
\inf _{s \in[0,1]} W^{\prime \prime}(u(s)) \leq W^{\prime \prime}(M) \leq \sup _{s \in[0,1]} W^{\prime \prime}(u(s))
$$

No matter what the value of $k,(58)$ and (59) together give a lower bound on the amplitude of $u$, which does not go to 0 as $\varepsilon \rightarrow 0$. The closer $M$ is to the center of the spinodal region, the better that bound is. Also, since $W^{\prime \prime}$ is bounded below, the first estimate in (58) will give a contradiction for $k$ sufficiently large. In fact, if $M$ is sufficiently close to the center (and $\varepsilon$ is not too large) it will give a contradiction for any $k>1$, so, in this case, each relevant equilibrium $u$ has exactly the same minimal period as the fastest-growing mode.

In any case, the calculations of this section have shown that all $\omega$-limit points of nonzero points on the pseudo-unstable manifold are equilibria with period $O(\varepsilon)$ and amplitude bounded away from 0. Anything close to one of these equilibria in the $L^{\infty}$ norm (a fortiori in the $H^{2}$ norm) will exhibit the small-wavelength large-amplitude oscillations characteristic of the spinodal decomposition observed in experiments.

\subsection{Orbits Approaching the $\omega$-limit Points}

In the previous two sections, the foundation has been laid for the application of the abstract results in Section 3 to the Cahn-Hilliard equation to show the predominance of spinodal decomposition. The following theorems are now immediate consequences of Corollary 2, Theorem 3, and Theorem 5. Several of the hypotheses are essentially the same for all theorems, so they will first be listed separately.

Let $M$ lie in the spinodal region of $W$, and let $\cos n_{0} \pi x$ be the unique fastest-growing mode of the linearization of the one-dimensional Cahn-Hilliard equation about $M$. Consider the linear manifold consisting of all $H^{2}$ functions on $[0,1]$ that have average value $M$ and satisfy Neumann boundary conditions. Let $\Omega$ be any neighborhood (in this manifold) of the collection of equilibria of the Cahn-Hilliard equation having average value $M$ and minimal period of the form $2 /\left(k n_{0}\right)$ for some positive integer $k$. Let $\Omega^{\prime}$ consist of all initial data that produce orbits entering $\Omega$ in positive time.

Theorem 6 Let $u_{0}$ be any point on the linear manifold of functions with mass $M$ that is not $H^{2}$-orthogonal to $\cos n_{0} \pi x$. If $|\rho|$ is sufficiently small then the 
solution of the Cahn-Hilliard equation with initial data $M+\rho\left(u_{0}-M\right)$ enters $\Omega$ in positive time; i.e., $M+\rho\left(u_{0}-M\right) \in \Omega^{\prime}$.

Theorem 7 Let $P$ be the projection defined by

$$
P\left(M+\sum_{n=0}^{\infty} a_{n} \cos n \pi x\right)=M+a_{n_{0}} \cos n_{0} \pi x .
$$

Let $\lambda_{n_{0}}$ be the growth rate of the fastest-growing mode of the linearization about $u \equiv M$ (as described in Section 2), and let $\lambda_{n_{1}}$ be the growth rate of the next fastest-growing mode. Then if

$$
p<\min \left\{\frac{\lambda_{n_{0}}}{\lambda_{n_{1}}}, 2\right\},
$$

there exist $r>0$ and $C>0$ such that if

$$
C\left\|u_{0}-P u_{0}\right\|_{H^{2}}^{p}<\left\|P u_{0}-M\right\|_{H^{2}}<r
$$

then $u_{0} \in \Omega^{\prime}$.

Theorem 8 Let $\varphi$ be any Gaussian measure of mean $M$ on the manifold of mass $M$ (i.e., a measure induced by a Gaussian measure of mean 0 on the parallel linear space) such that the induced distribution of $\left\langle\cdot, \cos n_{0} \pi x\right\rangle$ has nonzero variance. Let $\varphi_{\delta}$ be the scaled Gaussian measure on the manifold defined by

$$
\varphi_{\delta}(E)=\varphi\left(\frac{1}{\delta}(E-M)+M\right)
$$

for any Borel set E contained in the manifold. If $p$ is as in Theorem 7 then

$$
\varphi_{\delta}\left(\Omega^{\prime}\right)=1-O\left(\delta^{p-1}\right)
$$

as $\delta \rightarrow 0$.

All of these theorems are precise ways of stating that most choices of initial data near a constant in the spinodal region produce solutions that eventually exhibit spinodal decomposition.

\subsection{Generalizations and Nongeneric Cases}

As was shown above, the abstract results from Section 3 can be applied to the Cahn-Hilliard equation on any domain $\Omega$ such that $\partial \Omega$ is sufficiently smooth and $\operatorname{dim}(\Omega) \leq 3$. The difficulty is in obtaining sufficient information about 
the $\omega$-limit sets of the points on the pseudo-unstable manifold. Suppose, for example, that

$$
\Omega=\left[0, x_{0}\right] \times\left[0, y_{0}\right] \subset \mathbf{R}^{2} .
$$

The linearization of (1) about a constant $M$ in the spinodal region is

$$
\begin{array}{rl}
u_{t}=-\varepsilon^{2}\left(u_{x x x x}+2 u_{x x y y}+u_{y y y y}\right)-\beta^{2}\left(u_{x x}+u_{y y}\right) & (x, y) \in \Omega \\
u_{x}=u_{x x x}+u_{y y x}=0 & x \in\left\{0, x_{0}\right\} \\
u_{y}=u_{y y y}+u_{x x y}=0 & y \in\left\{0, y_{0}\right\} .
\end{array}
$$

Substituting

$$
u=a_{n, m} \cos \frac{n \pi x}{x_{0}} \cos \frac{m \pi y}{y_{0}}
$$

a typical eigenfunction of the Laplacian on $\Omega$, into (60) yields

$$
a_{n, m}(t)=a_{n, m}(0) \exp \left(\lambda_{n, m} t\right)
$$

where

$$
\lambda_{n, m}=r_{n, m}\left(\beta^{2}-\varepsilon^{2} r_{n, m}\right),
$$

and

$$
r_{n, m}=\left(\frac{n \pi}{x_{0}}\right)^{2}+\left(\frac{m \pi}{y_{0}}\right)^{2}=\pi^{2}\left(\frac{n^{2}}{x_{0}^{2}}+\frac{m^{2}}{y_{0}^{2}}\right) .
$$

If $x_{0}^{2} / y_{0}^{2}$ is irrational then, as when $\Omega=[0,1]$, for all but a discrete set of $\varepsilon$, there will be a unique fastest-growing mode for (60). Also, because the Cahn-Hilliard equation has symmetry-preserving properties on rectangles analogous to those on intervals, the relevant $\omega$-limit points will all have a smallwavelength periodic structure with characteristic length scale $O(\varepsilon)$. However, some information on the amplitude of the $\omega$-limit points must be obtained in order for the abstract results to imply spinodal decomposition. It is not obvious that these amplitudes could not be arbitrarily small, and if that happens then the appearance of spinodal decomposition has not been justified, since solutions with small amplitude have not decomposed at all.

If $x_{0}^{2} / y_{0}^{2}$ is rational then the set of $\varepsilon$ for which the pseudo-unstable manifold is one-dimensional may no longer be everywhere dense. When the pseudounstable manifold and subspace are of higher dimension then the smallest subspace that is invariant under (1) and contains the pseudo-unstable subspace is, in general, the entire space. Thus, for such $\varepsilon$ the $\omega$-limit points may not be periodic with small wavelength. The same problem occurs at a discrete set of $\varepsilon$ in the one-dimensional case. It may be possible to explain the appearance of spinodal decomposition in these pathological cases by some other methods, such as phase plane analysis on the pseudo-unstable manifold. 


\section{ACKNOWLEDGMENTS}

I wish to acknowledge the great help of Professors Paul Fife and Peter Bates. This research was supported in part by a National Science Foundation Graduate Fellowship and by the Institute for Mathematics and its Applications with funds provided by the National Science Foundation.

\section{References}

[1] P. W. Bates and C. K. R. T. Jones, Invariant manifolds for semilinear partial differential equations, in Dynamics Reported, Volume 2, U. Kirchgraber and H. O. Walther, eds., John Wiley and Sons, 1989, ch. 1, pp. 138.

[2] J. W. Cahn, On spinodal decomposition, Acta Metallurgica, 9 (1961), pp. $795-801$.

[3] J. W. Cahn, Phase separation by spinodal decomposition in isotropic systems, J. Chem. Phys., $\underline{42}$ (1965), pp. 93-99.

[4] J. W. Cahn, Spinodal decomposition, Transactions of the Metallurgical Society of AIME, $\underline{242}$ (1968), pp. 166-180.

[5] J. W. Cahn and J. E. Hilliard, Free energy of a nonuniform system I. interfacial free energy, Journal of Chemistry and Physics, $\underline{28}$ (1958), pp. 258-267.

[6] J. Carr, M. E. Gurtin, and M. Slemrod, Structured phase transitions on a finite interval, Arch. Rational Mech. Anal., $\underline{86}$ (1984), pp. 317-351.

[7] C. M. Elliott and S. Zheng, On the Cahn-Hilliard equation, Arch. Rational Mech. Anal., 96 (1986), pp. 339-357.

[8] D. Gilbarg and N. S. Trudinger, Elliptic Partial Differential Equations of Second Order, vol. 224 of Grundlehren der mathematischen Wissenschaften, Springer-Verlag, New York, 1983.

[9] J. K. Hale, Asymptotic Behavior of Dissipative Systems, vol. 25 of Mathematical Surveys and Monographs, American Mathematical Society, Providence, 1988.

[10] P. Hartman, Ordinary Differential Equations, Birkhäuser, Boston, second ed., 1982. 
[11] D. Henry, Geometric Theory of Semilinear Parabolic Equations, vol. 840 of Lecture Notes in Mathematics, Springer-Verlag, New York, 1981.

[12] M. W. Hirsch, C. C. Pugh, and M. Shub, Invariant Manifolds, vol. 583 of Lecture Notes in Mathematics, Springer-Verlag, New York, 1977.

[13] H.-H. Kuo, Gaussian Measures in Banach Spaces, vol. 463 of Lecture Notes in Mathematics, Springer-Verlag, New York, 1975.

[14] K. Lu, A Hartman-Grobman theorem for scalar reaction-diffusion equations, Tech. Rep. 686, Institute for Mathematics and its Applications, Sept. 1990.

[15] L. Modica, The gradient theory of phase transitions and the minimal interface criterion, Arch. Rational Mech. Anal., 98 (1987), pp. 123-142.

[16] J. R. Munkres, Topology: A First Course, Prentice-Hall, Englewood Cliffs, New Jersey, 1975.

[17] B. Nicolaenko and B. Scheurer, Low dimensional behaviour of the pattern formation Cahn-Hilliard equation, in Trends and Practice of Nonlinear Analysis, Lakshimikantham, ed., North Holland, 1985.

[18] A. Novick-Cohen and L. A. Segel, Nonlinear aspects of the Cahn-Hilliard equation, Phys. D, 10 (1984), pp. 277-298.

[19] A. Pazy, Semigroups of Linear Operators and Applications to Partial Differential Equations, vol. 44 of Applied Mathematical Sciences, SpringerVerlag, New York, 1983.

[20] S. M. Rankin, III, Semilinear evolution equations in Banach spaces with application to parabolic partial differential equations. Trans. Amer. Math. Soc., to appear.

[21] W. Rudin, Real and Complex Analysis, McGraw-Hill, New York, third ed., 1987.

[22] A. Skorohod, Integration in Hilbert Space, vol. 79 of Ergebnisse der Mathematik und ihrer Grenzgebiete, Springer-Verlag, New York, 1974.

[23] R. Temam, Infinite-Dimensional Dynamical Systems in Mechanics and Physics, vol. 68 of Applied Mathematical Sciences, Springer-Verlag, New York, 1988. 
[24] J. D. van der Waals, The thermodynamic theory of capillarity flow under the hypothesis of a continuous variation in density, Verhandelingen der Koninklijke Nederlandsche Akademie van Wetenschappen te Amsterdam, 1 (1893), pp. 1-56.

[25] S. Zheng, Asymptotic behavior of solution to the Cahn-Hilliard equation, Appl. Anal., $\underline{23}$ (1986), pp. 165-184. 